\title{
Application of the Sustainable Infrastructure Rating System for Developing Countries (SIRSDEC) to a case study
}

\author{
Jose Manuel Diaz-Sarachaga, Daniel Jato-Espino, Daniel Castro-Fresno* \\ GITECO Research Group, Universidad de Cantabria, Avd. de los Castros s/n, 39005 Santander (Spain)
}

E-mail addresses: jose-manuel.diazs@alumnos.unican.es (J.M. Diaz-Sarachaga); daniel.jato@unican.es (D. Jato-Espino); daniel.castro@unican.es (D. Castro-Fresno)

* Corresponding Author: Tel: +34 942 201550; Fax: +34 942201703

\begin{abstract}
A large amount of international public and private not-for-profit organizations strives to enhance the conditions of less developed economies under the flagship of sustainability throughout a wide range of infrastructure projects. However, the results are uncertain. Sustainable development in poorer countries requires effective frameworks to ensure the balanced consideration of social, economic and environmental dimensions. This paper discusses the application of the Sustainable Infrastructure Rating System for Developing Countries (SIRSDEC) to a mining infrastructure project located in Peru, in order to validate the methodology developed for this framework. The opinions returned from a questionnaire addressed to international experts according to the pairwise comparison scale of the Analytic Hierarchy Process (AHP) method were processed to obtain the weights of the elements forming the decision-making tree of SIRSDEC. The Integrated Value Model for Sustainable Assessment (MIVES) was introduced to assess infrastructure projects through the definition of value functions for each sustainability indicator, which enables the integration of variables measured in different units into a standardized value index. The weights obtained for SIRSDEC reflected the balance of the three pillars of sustainability, with a slight predominance of the social dimension. The case study highlighted the contribution of the new system to identify key sustainability issues which were omitted in the original project and posed several actions to improve community's perception and facilitate the development of the project.
\end{abstract}

\section{Keywords}

Sustainability; Developing countries; Rating System; SIRSDEC; AHP; MIVES.

\section{Introduction}

This article complements the structured methodology for creating the Sustainable Infrastructure Rating System for Developing Countries (SIRSDEC) (Diaz-Sarachaga et al., 2016a) as a decisive response to the urgent need to implement effective frameworks to support the principles of sustainable development worldwide. Existing rating tools for infrastructure do not involve a balanced consideration of social, economic and environmental aspects in the application of sustainability principles in these nations. SIRSDEC emphasizes the role of social and economic issues as a priority for the achievement of sustainable development goals (Gibberd, 2005), because less developed economies cannot be focused on environmental concerns (Libovich, 2005). Furthermore, management has been included as an additional dimension in this framework, in order to ensure that international standards and best practices are also taken into account as key guidelines to foster sustainability (Hiremath et al., 2013).

According to Belton and Steward (Belton et al., 2002), Multi-Criteria Decision Method (MCDM) is an umbrella term to describe a collection of formal approaches which seek to take explicit account of multiple criteria in helping individuals or groups to explore decisions that matter. The application of MCDMs provide decision-makers with effective frameworks to confidently select the most suitable options and rank alternatives from best to worst (Greco et al., 2005). The Analytic Hierarchy Process (AHP) (Saaty, 1980) is used in this article due to its simplicity and flexibility to be combined 
with other MCDMs such as the Integrated Value Model for Sustainable Assessment (MIVES) (ETCG, 2015), which enables standardizing different attributed indicators to easily compare a series of alternatives through a value index.

\section{SIRSDEC development framework}

\subsection{SIRSDEC decision-making tree}

SIRSDEC is an overarching framework that appraises the contribution to sustainability of infrastructure projects throughout their design, construction, operation, renovation and demolition/reuse stages. In accordance with the MIVES method, a decision-making tree was designed to structure SIRSDEC according to three hierarchical levels including 4 requirements, 23 criteria and 29 indicators (see Table 1). The requirements were related to the three pillars of sustainability (society, environment and economy) and management concerns, whilst the set of criteria and indicators derived from them were mostly selected after considering the objectives of Agenda 21 (UN, 1992), the Millennium Development Goals (MDGs) established in the United Nations (UN) Millennium Declaration (UN, 2000) and the Sustainable Development Goals (SDGs) adopted by the UN General Assembly on 25 September 2015 (UN, 2015), which are the main guidelines for sustainable development worldwide. The set of 29 indicators included in SIRSDEC are graded in a range from 0 to 1 point each, according to the value functions assigned to them. If all 29 indicators are rewarded with 1 point, the maximum possible SIRSDEC score is 121. Hence, SIRSDEC differentiates three levels of performance: Pass (63), Silver (from 63 to 90) and Gold (>90).

Table 1. SIRSDEC decision-making tree

\begin{tabular}{|c|c|c|c|c|c|}
\hline R\# & Requirement & C \#.\# & Criteria & I \#.\#.\# & Indicator \\
\hline \multirow{7}{*}{ R1 } & \multirow{7}{*}{ Management } & C 1.1 & International Standards & 1.1 .2 & $\begin{array}{l}\text { ISO } 9001 \text { or equivalent } \\
\text { ISO } 14001 \text { or equivalent }\end{array}$ \\
\hline & & C 1.2 & Project Sustainability Management (PSM) plan & 1.2 .1 & Project Sustainability Management plan \\
\hline & & C 1.3 & Sustainability Risk Management (SRM) plan & 1.3 .1 & Sustainability Risk Management plan \\
\hline & & C 1.4 & Sustainable Procurement plan & | 1.4 .1 & Sustainable Procurement plan \\
\hline & & C 1.5 & Inspection \& Auditing (I\&A) plan & | 1.5 .1 & I\&A plan \\
\hline & & 010 & & | 1.6 .1 & Periodic reports distribution \\
\hline & & C 1.6 & Reporting \& Lessons Learned (R\&LL) & | 1.6 .2 & Lessons Learned Log \\
\hline \multirow{6}{*}{ R2 } & \multirow{6}{*}{ Society } & C 2.1 & Community \& Stakeholders involvement & I 2.1.1 & Stakeholders involvement ratio \\
\hline & & C 2.2 & Role of indigenous people and communities & | 2.2 .1 & Indigenous involvement ratio \\
\hline & & C 2.3 & Equitable development & | 2.3.1 & Gender average wage ratio (female/male) \\
\hline & & & Social imnacts \& henefitc & | 2.4 .1 & Population impacted by project \\
\hline & & C 2.4 & Social impacts \& benefits & | 2.4 .2 & Settlements area disturbed \\
\hline & & C 2.5 & Cultural Heritage & | 2.5 .1 & Local cultural assessment \\
\hline \multirow{11}{*}{ R3 } & \multirow{11}{*}{ Environment } & C 3.1 & Natural Ecosystems conservation & I 3.1.1 & Impacted ecosystem area ratio \\
\hline & & C 3.2 & Biodiversity Ecosystem & | 3.2 .1 & Endangered species ratio \\
\hline & & & Greenhouse gases emissions & | 3.3 .1 & GHG emissions reduction rate \\
\hline & & & & | 3.4 .1 & Energy savings rate \\
\hline & & & 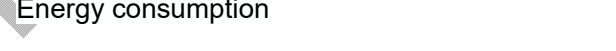 & | 3.4 .2 & Renewable energy use rate \\
\hline & & 35 & & | 3.5 .1 & Fresh water consumption reduction \\
\hline & & C 3.5 & Water management & | 3.5 .2 & Runoff water stored \\
\hline & & C 3.6 & Flooding risk & | 3.6 .1 & Floodplains area \\
\hline & & C 3.7 & Air Quality & | 3.7 .1 & Air pollutants reduction \\
\hline & & 38 & & | 3.8 .1 & Waste production decrease \\
\hline & & C 3.8 & Waste management & | 3.8 .2 & Recycled/reused waste \\
\hline \multirow{4}{*}{ R4 } & \multirow{4}{*}{ Economy } & C 4.1 & Combating poverty & I 4.1.1 & Local economic assessment \\
\hline & & C 4.2 & Agriculture impacts & | 4.2 .1 & Farmland area impacted \\
\hline & & C 4.3 & Local materials consumption & | 4.3.1 & Local materials use rate \\
\hline & & C 4.4 & Local employment & | 4.4.1 & Local employment rate \\
\hline
\end{tabular}

\subsection{Analysis of questionnaires}

An on-line questionnaire using Google Forms was addressed to 118 experts in the field of environmental and sustainable development, including professionals from public and private sectors such as development institutions, academia 
and industry. Expert participation is a key element for developing a weighting system to be incorporated into a sustainable assessment method (Chandratilake et al., 2013). The survey was conducted over the entire month of January 2016. 24 questionnaires were returned from experts belonging to 12 different countries as shown in Figure 1, which involves a response rate of $20.3 \%$. There were no invalid answers because the questionnaire format forced experts to reply all questions linked to the pairwise comparisons among the requirements, criteria and indicators of SIRSDEC according to the AHP scale (see Table 2). All these respondents had been involved in sustainability-driven projects and are aware of sustainable frameworks. 23 of them had worked with sustainable rating tools, whilst only one had no experience in this matter. 11 respondents $(45.8 \%)$ were academics, consultant and public sectors were represented by 5 participants $(20.8 \%)$ each and 3 experts $(12.6 \%)$ belonged to the contractor industry. The set of entities to which these experts were related are listed below:

BHP Billiton, Boluda Shipping Corporation and CWG Metro Riyad Joint Venture (Contractor industry); Waterloo University, Malaysia University Technology, Hamad Bin Khalifa University, University of Wisconsin-Madison, Coventry University, Colorado State University and Cardiff University (Academy); Qatar Green Council, AMEC Foster Wheeler, Tecnalia and Atkins (Consultancy); United Nations Office for Project Services (UNOPS), Agencia Española para Cooperación y Desarrollo (AECID), Qatar Foundation, International Organization of Supreme Audit Institutions Working Group on Environmental Auditing (INTOSAI WGEA) (Public development institutions).

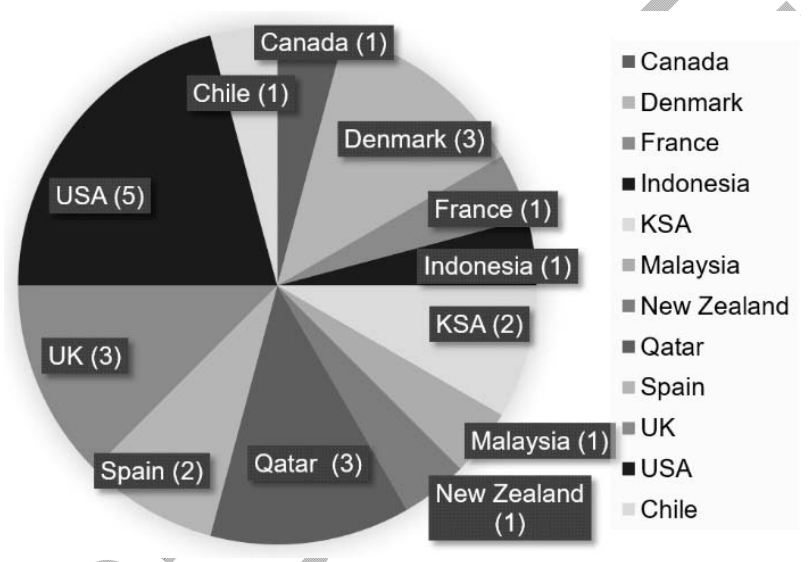

Figure 1. Countries of origin of the experts who responded to the questionnaire

Table 2. AHP pairwise comparison scale

\begin{tabular}{llc}
\hline Qualitative evaluation & & Rating \\
\hline Absolutely more important & $(\mathrm{AMI})$ & 9 \\
Much more important & $(\mathrm{MMI})$ & 7 \\
More important & $(\mathrm{MI})$ & 5 \\
Slightly more important & $(\mathrm{SMI})$ & 3 \\
Equally important & $(\mathrm{EI})$ & 1 \\
Slightly less important & $(\mathrm{SLI})$ & $1 / 3$ \\
Less important & $(\mathrm{LI})$ & $1 / 5$ \\
Much less important & $(\mathrm{MLI})$ & $1 / 7$ \\
Absolutely less important & $(\mathrm{ALI})$ & $1 / 9$
\end{tabular}

The survey also included three general questions to compare SIRSDEC with existing rating tools for infrastructures. 22 respondents $(91.7 \%)$ considered a sustainable infrastructure rating system focused on developing countries an effective framework for guiding development projects. 15 respondents $(62.5 \%)$ thought that the three sustainable principles should be equally weighted in the design process of a rating system for developing countries. The addition of management aspects to the sustainable Triple Bottom Line (TBL) was supported by 19 respondents $(79.2 \%)$. Besides, the participants were invited to propose the removal and/or addition of some criteria and/or indicators to SIRSDEC. Disaster risk reduction for resilience, noise pollution plans and the consideration of the relationships with authorities were the three additional criteria suggested by the experts. Two new indicators were also proposed to be incorporated into the system: amount of land cleared and embodied energy in built infrastructure. Finally, some respondents showed their 
preference to discard C1.3, I1.1.1 and I1.3.1. Since these parameters belong to the management requirement, which is deemed to be the fourth pillar of sustainability, they remained in the SIRSDEC decision-making tree to emphasize the role of this dimension. Table 3 shows the linguistic comparisons (see Table 2) provided by the experts with respect to 112 each pair of elements in the decision-making tree. 
113

114
115

Table 3. Experts' judgments according to the elements in the decision-making tree

\begin{tabular}{|c|c|c|c|c|c|c|c|c|c|c|c|c|c|c|c|c|c|c|c|c|c|c|c|c|}
\hline $\begin{array}{l}\text { Parwwise } \\
\text { Comparison }\end{array}$ & \multicolumn{24}{|c|}{ Expert vumber } \\
\hline $\begin{array}{ll}\text { R1 vs R2 } \\
\text { R }\end{array}$ & SLI & & MLI & EI & & SMI & & EI & $\begin{array}{l}\text { SLI } \\
\text { SI }\end{array}$ & El & MI & $\mathrm{EI}_{\mathrm{E}}$ & SLI & SLI & LI & SLI & 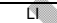 & EI & SET & EI & & & MLI & SMI \\
\hline 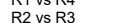 & . & & & 年 & 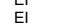 & & 年 & 促 & & & EI & 促 & 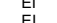 & 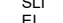 & El & & & & SLI & SLI & MLI & SMI & MLI & \\
\hline 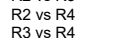 & EI & Mi & $\begin{array}{l}\mathrm{MI} \\
\mathrm{MI}\end{array}$ & EI & EI & $\begin{array}{ll}\mathrm{L} \\
\mathrm{MI}\end{array}$ & $\begin{array}{l}\text { SLI } \\
\end{array}$ & EI & EI & $\begin{array}{l}\mathrm{El} \\
\mathrm{EI}\end{array}$ & $\underset{\substack{E I \\
\text { EI }}}{E \text { I }}$ & $\begin{array}{l}\text { SMI } \\
\text { SL }\end{array}$ & SMI & $\begin{array}{l}\text { SMI } \\
\text { SMI }\end{array}$ & SMI & $\begin{array}{l}\text { Sul } \\
\text { Sul }\end{array}$ & $\begin{array}{l}\text { SMI } \\
\text { SMI }\end{array}$ & & EI & SMI & SMI & MMI & $\begin{array}{l}\text { EI } \\
\text { EI } \\
\text { EI }\end{array}$ & $\begin{array}{l}E I \\
\text { EI } \\
\text { EI }\end{array}$ \\
\hline 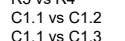 & $\begin{array}{l}\text { SLI } \\
\text { sL }\end{array}$ & $\begin{array}{l}\text { MII } \\
\text { SLI } \\
\text { MLI }\end{array}$ & $\begin{array}{l}\text { MI } \\
\text { EI } \\
\text { SII }\end{array}$ & $\begin{array}{l}\text { SMI } \\
\text { SMI }\end{array}$ & $\begin{array}{l}E I \\
\text { MLI } \\
\text { MLI }\end{array}$ & $\begin{array}{l}\text { MI } \\
\text { EI } \\
\text { EI }\end{array}$ & MI & LI & 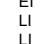 & $\begin{array}{l}\mathrm{EI} \\
\mathrm{LI} \\
\mathrm{SL}\end{array}$ & SMI & $\begin{array}{l}\text { SII } \\
\text { LIMI }\end{array}$ & $\begin{array}{l}\text { MI } \\
\text { EI } \\
\text { 丩I }\end{array}$ & SMI & SLI & $\begin{array}{l}\mathrm{S} \\
\mathrm{E} \\
\mathrm{E}\end{array}$ & $\begin{array}{l}\text { SMI } \\
\text { MI }\end{array}$ & $\begin{array}{l}\text { EI } \\
\text { LI }\end{array}$ & $\begin{array}{l}\mathrm{EI} \\
\mathrm{LI} \\
\mathrm{LI}\end{array}$ & $\begin{array}{l}\mathrm{EI} \\
\mathrm{EI} \\
\mathrm{H}\end{array}$ & $\begin{array}{l}\text { MI } \\
\text { ALI }\end{array}$ & $\begin{array}{l}\text { MI } \\
\text { EI }\end{array}$ & 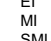 & MMI \\
\hline 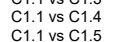 & $\begin{array}{l}\text { SLI } \\
\text { SLI }\end{array}$ & $\begin{array}{l}\text { MLI } \\
\text { MLL } \\
\text { MLI }\end{array}$ & $\begin{array}{l}\text { SMI } \\
\text { MLI }\end{array}$ & $\begin{array}{l}\text { SMI } \\
\text { SMI }\end{array}$ & $\begin{array}{l}\text { MLI } \\
\text { ALI }\end{array}$ & $\begin{array}{l}\mathrm{EI} \\
\mathrm{MI} \\
\mathrm{EI}\end{array}$ & $\begin{array}{l}\mathrm{MII} \\
\mathrm{MI}\end{array}$ & $\begin{array}{l}\text { AMI } \\
\text { MII }\end{array}$ & $\begin{array}{l}\mathrm{LI} \\
\mathrm{LI}\end{array}$ & $\begin{array}{l}\text { ALI } \\
\text { ALI }\end{array}$ & $\begin{array}{l}\mathrm{LI} \\
\mathrm{EI} \\
\mathrm{MI}\end{array}$ & $\begin{array}{l}\text { SLII } \\
\text { SLI }\end{array}$ & $\begin{array}{l}L 1 \\
\text { LI } \\
\text { EI }\end{array}$ & $\begin{array}{l}\text { LI } \\
\text { L }\end{array}$ & $\begin{array}{l}\mathrm{MI} \\
\mathrm{EI} \\
\mathrm{SMI}\end{array}$ & $\begin{array}{ll}E \\
E \\
\text { EI } \\
\text { EI }\end{array}$ & $\begin{array}{l}\mathrm{MII} \\
\mathrm{MI} \\
\mathrm{LI}\end{array}$ & $\begin{array}{l}\text { SMI } \\
\text { SLI }\end{array}$ & $\begin{array}{l}L 1 \\
\stackrel{L}{S L I}\end{array}$ & SMI & $\begin{array}{l}\text { ALI } \\
\text { ALI }\end{array}$ & $M_{E I}^{M M I}$ & MII & $\begin{array}{l}\text { MMI } \\
\text { SMI }\end{array}$ \\
\hline $\begin{array}{l}\text { C1.1.1 v s C } 1.6 \\
\text { C1.2 vs } C 1.3\end{array}$ & SLI & $\begin{array}{l}\text { MLI } \\
\text { EI }\end{array}$ & $\begin{array}{ll}\mathrm{L} \\
\mathrm{L} \\
\mathrm{MI}\end{array}$ & $\begin{array}{l}\text { SMI } \\
\text { EI }\end{array}$ & $\begin{array}{l}\text { MLI } \\
\text { MI }\end{array}$ & MII & $\begin{array}{ll}\mathrm{Ml} \\
\mathrm{MI}\end{array}$ & $\begin{array}{l}L \\
\text { SLI } \\
\text { SLI }\end{array}$ & $\begin{array}{l}\text { LI } \\
\text { SMI }\end{array}$ & LI & $\begin{array}{l}\text { MII } \\
\text { MII }\end{array}$ & $\begin{array}{l}\text { SMI } \\
\text { SML }\end{array}$ & $\begin{array}{ll}L \\
\text { LI } \\
\text { EI }\end{array}$ & SLI & $\begin{array}{l}\text { EI } \\
\text { SIMI }\end{array}$ & SMI & ST & MI & $\begin{array}{ll}\mathrm{El} \\
\mathrm{El}\end{array}$ & $\begin{array}{l}\text { SLI } \\
\text { SLI }\end{array}$ & $\begin{array}{l}\text { ALI } \\
\text { ALI }\end{array}$ & MMI & $\begin{array}{l}\text { SWI } \\
\text { EI }\end{array}$ & $\begin{array}{l}\text { MI } \\
\text { SMI }\end{array}$ \\
\hline 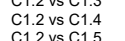 & EI & EI & MMI & EI & $\begin{array}{l}\text { EI } \\
\text { EI } \\
\end{array}$ & $\begin{array}{l}\text { sMI } \\
\text { SII }\end{array}$ & $\begin{array}{l}\text { MI } \\
\text { LI }\end{array}$ & SLI & SMI & 列 & EI & SLI & MMI & $\begin{array}{l}\text { SLI } \\
\text { SII }\end{array}$ & EI & EI & Mil & $\begin{array}{l}\text { SMI } \\
\text { SMI }\end{array}$ & EI & $\begin{array}{l}\text { SMI } \\
\text { SMI }\end{array}$ & EI & MMI & $\begin{array}{l}\text { SLI } \\
\text { SMI }\end{array}$ & SMI \\
\hline $\begin{array}{l}\text { C1.2.2 v v C1.6 } \\
\text { c1.3vs C1. }\end{array}$ & $\begin{array}{l}\text { EI } \\
\text { EI } \\
\text { EI }\end{array}$ & $\begin{array}{l}\mathrm{El} \\
\mathrm{EI} \\
\mathrm{EI}\end{array}$ & $\begin{array}{l}\text { EI } \\
\text { SMI } \\
\text { MI }\end{array}$ & $\begin{array}{l}\text { MI } \\
\text { EI } \\
\text { SLI }\end{array}$ & $\begin{array}{l}L \\
\text { LI } \\
\text { SLI }\end{array}$ & $\begin{array}{l}\mathrm{EI} \\
\mathrm{E} \\
\mathrm{EI}\end{array}$ & $\begin{array}{l}\mathrm{MI} \\
\mathrm{MI} \\
\text { SMI }\end{array}$ & $\begin{array}{l}\mathrm{SLI} \\
\mathrm{EI}\end{array}$ & $\begin{array}{l}\text { EI } \\
\text { SMI } \\
\text { SMI }\end{array}$ & $\begin{array}{l}E I \\
\text { EI } \\
\text { EI }\end{array}$ & $\begin{array}{l}M I \\
M I \\
E I\end{array}$ & $\begin{array}{l}\text { SIII } \\
\text { ELI }\end{array}$ & $\begin{array}{l}\text { MMI } \\
\text { SMII } \\
\text { MMI }\end{array}$ & $\begin{array}{l}\text { SII } \\
\text { EI }\end{array}$ & $\begin{array}{l}\mathrm{El} \\
\mathrm{MI} \\
\mathrm{El}\end{array}$ & $\begin{array}{l}E 1 \\
\text { EI } \\
E \text { EI }\end{array}$ & $\begin{array}{l}\mathrm{El} \\
\mathrm{EI} \\
\mathrm{SMI}\end{array}$ & $\begin{array}{l}\mathrm{EI} \\
\mathrm{MI} \\
\mathrm{SLI}\end{array}$ & $\begin{array}{l}E I \\
\mathrm{El} \\
\mathrm{SLI}\end{array}$ & $\begin{array}{l}\text { SMI } \\
\text { SMI }\end{array}$ & $\begin{array}{l}E I \\
M I \\
\text { EI }\end{array}$ & $\begin{array}{l}L 1 \\
\mathrm{EI} \\
\mathrm{SLI}\end{array}$ & $\begin{array}{l}\text { SLI } \\
\text { MI }\end{array}$ & $\begin{array}{l}\text { MII } \\
\text { SMI } \\
\text { SMI }\end{array}$ \\
\hline $\begin{array}{l}\text { C1.3v s s c1.5 } \\
\text { C1.3vs C1.6. }\end{array}$ & $\begin{array}{l}\text { EI } \\
\text { EI } \\
\text { EI }\end{array}$ & $\begin{array}{l}\mathrm{El} \\
\text { EI } \\
\text { SMI }\end{array}$ & $\begin{array}{l}\text { MI } \\
\text { EI } \\
\text { SLI }\end{array}$ & $\begin{array}{l}\text { SLI } \\
\text { SLI }\end{array}$ & $\stackrel{\mathrm{LI}}{\mathrm{L}}$ & $\begin{array}{l}E l \\
\text { El } \\
\text { EI }\end{array}$ & $\begin{array}{l}\text { SLI } \\
\mathrm{EI}\end{array}$ & $\begin{array}{l}E I \\
M I \\
E I\end{array}$ & $\begin{array}{l}\text { SLI } \\
\text { SLI }\end{array}$ & $\begin{array}{l}\mathrm{El} \\
\text { SLI } \\
\mathrm{EI}\end{array}$ & $\begin{array}{l}\mathrm{El} \\
\mathrm{El} \\
\mathrm{MI}\end{array}$ & $\begin{array}{l}\text { EI } \\
\text { EI } \\
\text { SMI }\end{array}$ & $\begin{array}{l}\text { MMI } \\
\text { MI } \\
\text { EI }\end{array}$ & $\begin{array}{l}\text { SLII } \\
\text { SLI }\end{array}$ & $\begin{array}{l}\text { EI } \\
\text { EI } \\
\text { LI }\end{array}$ & 皆 & $\begin{array}{l}\text { SMI } \\
\text { SLI }\end{array}$ & $\begin{array}{l}\text { LI } \\
\text { LLI }\end{array}$ & $\begin{array}{l}\text { SLI } \\
\text { SMI } \\
\text { EI }\end{array}$ & $\begin{array}{l}\text { SLI } \\
\text { SLI }\end{array}$ & $\begin{array}{l}\text { EI } \\
\text { SMI }\end{array}$ & MLI & $\begin{array}{l}\text { MII } \\
\text { SLI }\end{array}$ & $\begin{array}{l}\text { SMI } \\
\text { SMI }\end{array}$ \\
\hline $\begin{array}{l}\text { c1.4 v C } 1.5 \\
\text { C1.4 vs C1.6 }\end{array}$ & $\begin{array}{ll}\mathrm{EI} \\
\mathrm{EI}\end{array}$ & $\begin{array}{l}\mathrm{Ml} \\
\mathrm{El}\end{array}$ & $\begin{array}{l}\text { SLL } \\
\text { SLI }\end{array}$ & $\begin{array}{l}\text { SMI } \\
\mathrm{El}\end{array}$ & $\begin{array}{l}L \\
\text { LI }\end{array}$ & $\begin{array}{l}\mathrm{El} \\
\mathrm{El}\end{array}$ & $\begin{array}{l}\mathrm{SLI} \\
\mathrm{MI}\end{array}$ & $\begin{array}{l}\text { SLI } \\
\text { SLI }\end{array}$ & $\begin{array}{l}\mathrm{LI} \\
\mathrm{LI}\end{array}$ & $\begin{array}{l}\text { SMI } \\
\mathrm{EI}\end{array}$ & $\begin{array}{l}M 1 \\
\text { MI }\end{array}$ & $\begin{array}{l}\text { SMI } \\
\text { SLI }\end{array}$ & EI & $\begin{array}{l}\text { SLI } \\
\text { SLI }\end{array}$ & $\begin{array}{l}\mathrm{EI} \\
\mathrm{LI}\end{array}$ & $\begin{array}{l}\text { EI } \\
\text { EI } \\
\mathrm{El}\end{array}$ & $\stackrel{\mathrm{LI}}{\mathrm{LI}}$ & $\begin{array}{l}\mathrm{El} \\
\mathrm{EMI} \\
\text { SMI }\end{array}$ & $\begin{array}{c}\mathrm{EI} \\
\mathrm{EI} \\
\mathrm{EI}\end{array}$ & $\begin{array}{l}\text { SLI } \\
\text { SLL }\end{array}$ & $\begin{array}{l}\text { SMI } \\
\text { EI }\end{array}$ & $\begin{array}{ll}\mathrm{LL} \\
\mathrm{SLI}\end{array}$ & $\begin{array}{l}\mathrm{EI} \\
\mathrm{SLI}\end{array}$ & $\begin{array}{l}\text { SMI } \\
\text { SLI }\end{array}$ \\
\hline $\begin{array}{l}\text { c1.5 v c1.6. } \\
\text { C2.1 vs C2.2 }\end{array}$ & EI & $\begin{array}{l}\text { I } \\
\text { EI } \\
\text { SLI }\end{array}$ & $\begin{array}{l}\text { EI } \\
\text { SLI }\end{array}$ & $\begin{array}{l}\text { SLI } \\
\text { SLI }\end{array}$ & $\begin{array}{l}\text { EI } \\
\text { EI } \\
\text { El }\end{array}$ & $\begin{array}{l}\mathrm{L} \\
\mathrm{E} \mid \\
\mathrm{EI}\end{array}$ & $\begin{array}{l}\text { MI } \\
\text { MMI }\end{array}$ & $\begin{array}{l}\text { EI } \\
\text { SLI }\end{array}$ & $\begin{array}{l}\text { EI } \\
\text { SMI }\end{array}$ & $\begin{array}{l}\text { SLI } \\
\mathrm{LI}\end{array}$ & $\begin{array}{l}\mathrm{MI} \\
\mathrm{MI} \\
\mathrm{MI}\end{array}$ & $\begin{array}{l}\text { SMI } \\
\text { SLI }\end{array}$ & & $\begin{array}{l}\mathrm{SMI} \\
\mathrm{EI}\end{array}$ & MI & $\begin{array}{l}\mathrm{EI} \\
\mathrm{SLLI}\end{array}$ & El & $\begin{array}{l}\mathrm{EI} \\
\mathrm{EI}\end{array}$ & $\begin{array}{l}\text { SLI } \\
\text { SLI }\end{array}$ & $\begin{array}{l}\mathrm{EI} \\
\mathrm{EI}\end{array}$ & $\begin{array}{c}\mathrm{EI} \\
\mathrm{EI} \\
\mathrm{EI}\end{array}$ & $\begin{array}{l}\text { MLI } \\
\text { EI }\end{array}$ & $\begin{array}{c}\text { EI } \\
\text { EI } \\
\text { EI }\end{array}$ & $\begin{array}{l}\text { SMI } \\
\text { MI }\end{array}$ \\
\hline $\begin{array}{l}\text { C2.1 v C2.3 } \\
\text { C2.1 v C C2.4 }\end{array}$ & $\begin{array}{l}\text { SMI } \\
\text { SLI }\end{array}$ & SMI & $\begin{array}{l}\text { EI } \\
\text { SLI }\end{array}$ & $\begin{array}{l}\mathrm{EI} \\
\mathrm{EI}\end{array}$ & $\begin{array}{l}\mathrm{MI} \\
\mathrm{EI}\end{array}$ & $\begin{array}{l}\text { SMI } \\
\text { SMI }\end{array}$ & $\begin{array}{l}\text { SMI } \\
\text { SMI }\end{array}$ & $\begin{array}{l}\text { SLI } \\
\text { SLI }\end{array}$ & $\begin{array}{l}\text { SLI } \\
\mathrm{EI}\end{array}$ & $\underset{\mathrm{EI}}{\mathrm{LI}}$ & $\begin{array}{l}\text { MMI } \\
\text { MMI }\end{array}$ & $\begin{array}{l}\mathrm{El} \\
\mathrm{SLI}\end{array}$ & EI & & EI & $\begin{array}{l}\text { SLI } \\
\text { EII }\end{array}$ & $\begin{array}{l}\mathrm{EI} \\
\mathrm{SMI}\end{array}$ & EI & $\begin{array}{l}\text { SLI } \\
\text { SLI }\end{array}$ & $\begin{array}{l}\text { SLLI } \\
\text { SLI }\end{array}$ & EI & $\underset{\substack{\mathrm{EI} \\
\mathrm{EI}}}{\mathrm{EI}}$ & EI & $\underset{M I I}{M I I}$ \\
\hline 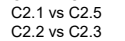 & $\underset{\mathrm{E}}{\mathrm{L}}$ & $\begin{array}{l}\text { SMI } \\
\text { MI }\end{array}$ & $\begin{array}{l}\text { SLI } \\
\text { MI }\end{array}$ & $\begin{array}{l}\text { SMI } \\
\text { SMI }\end{array}$ & EI & $\begin{array}{l}\text { SLI } \\
\text { EI }\end{array}$ & $\begin{array}{l}\mathrm{LI} \\
\mathrm{SLI}\end{array}$ & $\begin{array}{l}\text { SLI } \\
\text { AMI }\end{array}$ & $\begin{array}{l}\text { EII } \\
\text { SLI }\end{array}$ & $\begin{array}{l}S L I \\
\mathrm{EI}\end{array}$ & $\underset{E \mathrm{E}}{\mathrm{MMI}}$ & $\begin{array}{l}\text { SLI } \\
\text { SMII }\end{array}$ & $\begin{array}{l}\text { sut } \\
\text { st }\end{array}$ & & $\begin{array}{l}\text { EI } \\
\text { SMI }\end{array}$ & $\begin{array}{l}\mathrm{EI} \\
\text { SMI }\end{array}$ & $\underset{\mathrm{MI}}{\mathrm{MI}}$ & EI & $\begin{array}{l}\text { SLI } \\
\text { EI }\end{array}$ & $\begin{array}{l}\text { MLI } \\
\text { MI }\end{array}$ & $\begin{array}{l}\text { EI } \\
\text { EI }\end{array}$ & $\begin{array}{l}\mathrm{EI} \\
\mathrm{SLI}\end{array}$ & EI & $\underset{\mathrm{EI}}{\mathrm{MI}}$ \\
\hline $\begin{array}{l}\text { C2.2 v c2.4 } \\
\text { C2.2 vs C2.5 }\end{array}$ & $\begin{array}{l}\text { EI } \\
\text { EI }\end{array}$ & $\begin{array}{l}\mathrm{E} \text { II } \\
\text { MI }\end{array}$ & $\underset{E I}{M I}$ & $\begin{array}{l}\text { EI } \\
\text { SMI }\end{array}$ & $\underset{\mathrm{EI}}{\mathrm{MI}}$ & $\begin{array}{l}\text { SMI } \\
\mathrm{E} \text { EI }\end{array}$ & $\stackrel{\text { MLI }}{\text { MLI }}$ & $\begin{array}{l}\text { AMI } \\
\text { AMI }\end{array}$ & 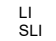 & $\underset{\substack{E I \\
\text { EI }}}{2}$ & $\begin{array}{l}\text { SMI } \\
\text { MI }\end{array}$ & $\begin{array}{l}\text { SMI } \\
\text { SLI }\end{array}$ & $\begin{array}{l}\text { EI } \\
\text { SLL. }\end{array}$ & $\begin{array}{l}\mathrm{LI} \\
\mathrm{SLI}\end{array}$ & $\begin{array}{c}\mathrm{EI} \\
\mathrm{EI}\end{array}$ & $\begin{array}{l}\text { SMI } \\
\text { SMII }\end{array}$ & $\begin{array}{l}\mathrm{EI} \\
\mathrm{LI}\end{array}$ & $\begin{array}{l}\text { EI } \\
\text { SMI }\end{array}$ & $\begin{array}{l}\text { EI } \\
\text { SMI }\end{array}$ & $\begin{array}{l}\text { SMI } \\
\text { EI }\end{array}$ & $\begin{array}{c}\mathrm{EI} \\
\mathrm{EI} \\
\mathrm{E}\end{array}$ & $\underset{\mathrm{EI}}{\mathrm{LI}}$ & $\begin{array}{c}\mathrm{EI} \\
\mathrm{EI}\end{array}$ & $\underset{\substack{\mathrm{EI} \\
\mathrm{EI}}}{ }$ \\
\hline 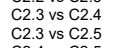 & $\begin{array}{l}\text { EI } \\
\text { EI }\end{array}$ & $\begin{array}{l}\text { SLI } \\
\text { SMI }\end{array}$ & $\begin{array}{l}\text { EI } \\
\text { EI }\end{array}$ & $\begin{array}{l}\text { EI } \\
\text { SMI }\end{array}$ & $\begin{array}{l}\text { EI } \\
\text { EI }\end{array}$ & $\begin{array}{l}\text { SMI } \\
\text { SLI }\end{array}$ & $\begin{array}{l}\text { SMI } \\
\text { SLI }\end{array}$ & $\begin{array}{l}\text { MMI } \\
\text { AMI }\end{array}$ & $\begin{array}{l}\text { EI } \\
\text { SMI }\end{array}$ & $\begin{array}{l}\text { SMI } \\
\text { MI }\end{array}$ & $\begin{array}{l}\text { MMI } \\
\text { MI }\end{array}$ & $\begin{array}{l}\text { SMII } \\
\text { SMI }\end{array}$ & $\begin{array}{l}\text { SMI } \\
\text { SMI }\end{array}$ & $\begin{array}{l}\text { SLI } \\
\text { SLI }\end{array}$ & $\begin{array}{l}\mathrm{LI} \\
\mathrm{E}\end{array}$ & $\begin{array}{l}\mathrm{El} \\
\mathrm{E} \mid \mathrm{I}\end{array}$ & $\begin{array}{l}\mathrm{EI} \\
\mathrm{MI}\end{array}$ & $\begin{array}{l}\text { EI } \\
\text { MI }\end{array}$ & $\begin{array}{l}\text { EI } \\
\text { SMI }\end{array}$ & $\begin{array}{l}\text { SMI } \\
\text { EI }\end{array}$ & $\stackrel{\stackrel{L}{L}}{\mathrm{~L}}$ & $\begin{array}{l}\text { SMI } \\
\mathrm{EI}\end{array}$ & $\begin{array}{l}\mathrm{Ml} \\
\mathrm{El}\end{array}$ & $\begin{array}{l}\text { SMI } \\
\mathrm{El}\end{array}$ \\
\hline $\begin{array}{l}\mathrm{C} 2.4 \mathrm{vs} C 2.5 \\
\mathrm{C} 3.1 \text { } \mathrm{C} C 3.2\end{array}$ & 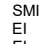 & $\begin{array}{l}\text { MI } \\
\mathrm{EI}\end{array}$ & $\begin{array}{l}\text { MI } \\
\mathrm{EI}\end{array}$ & $\begin{array}{l}\mathrm{EI} \\
\mathrm{EI}\end{array}$ & $\begin{array}{l}\text { El } \\
\text { EI }\end{array}$ & $\begin{array}{l}\text { EI } \\
\text { SMI }\end{array}$ & $\begin{array}{l}\text { SMI } \\
\text { MI }\end{array}$ & $\begin{array}{l}\mathrm{EI} \\
\mathrm{MMI}\end{array}$ & $\begin{array}{l}\mathrm{MI} \\
\mathrm{MI}\end{array}$ & $\begin{array}{l}\text { SMI } \\
\text { EI }\end{array}$ & $\begin{array}{l}\text { MMI } \\
\text { EI }\end{array}$ & $\begin{array}{l}\text { SLI } \\
\text { SMIt } \\
\text { SI }\end{array}$ & $\begin{array}{l}\text { SMI } \\
\text { MI }\end{array}$ & $\begin{array}{l}\text { SMI } \\
\mathrm{EI}\end{array}$ & $\begin{array}{l}\mathrm{EI} \\
\mathrm{EI}\end{array}$ & $\begin{array}{l}\text { EI } \\
\text { EI }\end{array}$ & $\begin{array}{l}\mathrm{MI} \\
\mathrm{EI}\end{array}$ & $\begin{array}{c}\mathrm{MI} \\
\mathrm{EI}\end{array}$ & $\begin{array}{l}\text { SMI } \\
\text { EI }\end{array}$ & $\begin{array}{l}\text { SLI } \\
\text { EI }\end{array}$ & $\begin{array}{l}\mathrm{EI} \\
\mathrm{EI}\end{array}$ & $\begin{array}{l}\mathrm{EI} \\
\mathrm{EI}\end{array}$ & $\begin{array}{c}E I \\
\text { EI } \\
\text { II }\end{array}$ & $\begin{array}{l}S M I \\
\mathrm{EI}\end{array}$ \\
\hline $\begin{array}{l}\text { C3.1 vs C3.3 } \\
\text { C3.1 vs } C 3.4\end{array}$ & $\begin{array}{l}\text { EI } \\
\text { EI }\end{array}$ & $\begin{array}{l}\text { SMI } \\
\text { SMI }\end{array}$ & $\begin{array}{l}\text { EI } \\
\text { MI }\end{array}$ & $\begin{array}{l}\text { EI } \\
\text { EI }\end{array}$ & $\begin{array}{l}\mathrm{MI} \\
\mathrm{MI}\end{array}$ & $\begin{array}{l}\text { 년 } \\
\text { LI }\end{array}$ & $\begin{array}{l}\text { MI } \\
\text { MMI }\end{array}$ & $\stackrel{\text { LM }}{\text { MI }}$ & $\begin{array}{l}\text { EI } \\
\text { EI }\end{array}$ & $\begin{array}{l}\text { EI } \\
\text { EI }\end{array}$ & $\begin{array}{l}\text { EI } \\
\text { EI }\end{array}$ & $\begin{array}{l}\text { SLI } \\
\text { SMI } \\
\text { SMI }\end{array}$ & $\begin{array}{l}\text { EI } \\
\text { SML }\end{array}$ & $\begin{array}{l}\text { SMI } \\
\text { SMI }\end{array}$ & $\begin{array}{l}\text { EI } \\
\text { MI }\end{array}$ & MII & $\begin{array}{l}\mathrm{EI} \\
\mathrm{EI}\end{array}$ & $\begin{array}{l}\mathrm{EI} \\
\mathrm{MI}\end{array}$ & $\begin{array}{l}\text { SMI } \\
\text { EI }\end{array}$ & $\begin{array}{l}\text { SMI } \\
\text { SMI }\end{array}$ & $\begin{array}{l}\mathrm{EI} \\
\mathrm{E} \mid\end{array}$ & $\begin{array}{l}\text { EI } \\
\text { MMI }\end{array}$ & $\begin{array}{l}\text { SLI } \\
\text { EI }\end{array}$ & $\begin{array}{l}\mathrm{EI} \\
\mathrm{MI}\end{array}$ \\
\hline $\begin{array}{l}\text { C3.1 v } 3.5 .5 \\
\text { C3.1 v C3.6 }\end{array}$ & $\begin{array}{l}\text { EI } \\
\text { EI } \\
\text { EI }\end{array}$ & $\begin{array}{l}\text { EI } \\
\text { SMI } \\
\text { SMI }\end{array}$ & $\begin{array}{l}\text { SMI } \\
\text { MMI } \\
\text { I }\end{array}$ & $\begin{array}{l}\text { EI } \\
\text { EI } \\
S \$ 1\end{array}$ & $\begin{array}{l}\mathrm{EI} \\
\mathrm{EI}\end{array}$ & 년 & $\begin{array}{l}\text { MMI } \\
\text { MI } \\
M\end{array}$ & $\begin{array}{l}\text { AMII } \\
\text { AMI }\end{array}$ & $\begin{array}{l}\text { EI } \\
\text { EI } \\
\text { EI }\end{array}$ & $\begin{array}{l}\text { EI } \\
\text { EI } \\
\text { EI }\end{array}$ & EI & $\begin{array}{l}\text { SMI } \\
\text { MI } \\
\text { SM }\end{array}$ & $\begin{array}{l}\text { SMI } \\
\text { MI }\end{array}$ & $\begin{array}{l}\text { SMI } \\
\text { SMII } \\
\text { SII }\end{array}$ & $\begin{array}{l}\mathrm{LI} \\
\mathrm{SLI}\end{array}$ & $\begin{array}{l}\text { SMI } \\
\text { SMII } \\
\text { SII }\end{array}$ & $\begin{array}{l}\text { EI } \\
\text { SMI } \\
\text { SI }\end{array}$ & $\begin{array}{l}\mathrm{MI} \\
\mathrm{EI}\end{array}$ & $\begin{array}{l}E I \\
\text { SLI }\end{array}$ & $\begin{array}{l}\text { SLI } \\
\text { SLI }\end{array}$ & $\begin{array}{l}E I \\
E I\end{array}$ & $\begin{array}{l}\text { EI } \\
\mathrm{LI}\end{array}$ & $\begin{array}{l}\text { SLI } \\
\text { SMI }\end{array}$ & $\begin{array}{l}\text { SMI } \\
\text { SMI }\end{array}$ \\
\hline $\begin{array}{l}\text { 3.1. vs c3.7 } \\
\text { C3.1 v c } 3.8\end{array}$ & $\begin{array}{l}\text { EI } \\
\text { EI }\end{array}$ & $\begin{array}{l}\text { SMI } \\
\text { SMI }\end{array}$ & $\begin{array}{l}\text { EI } \\
\text { SLI }\end{array}$ & $\begin{array}{l}\text { SLI } \\
\text { SLI }\end{array}$ & $\begin{array}{l}\text { MI } \\
\text { SMI }\end{array}$ & $\begin{array}{l}\text { L } \\
\stackrel{4}{L}\end{array}$ & $\begin{array}{l}\text { MI } \\
\text { MII }\end{array}$ & $\stackrel{\text { LI }}{\mathrm{L}}$ & $\begin{array}{l}\text { EI } \\
\text { EI } \\
\text { SI }\end{array}$ & $\begin{array}{l}\mathrm{EI} \\
\mathrm{EI} \\
\mathrm{SI}\end{array}$ & $M U$ & $\begin{array}{l}\text { SMI } \\
\text { MI }\end{array}$ & $\begin{array}{l}\text { SMI } \\
\text { SMI } \\
\text { SM }\end{array}$ & $\begin{array}{l}\text { SMI } \\
\text { SMII } \\
\text { SMI }\end{array}$ & $\begin{array}{l}\mathrm{EI} \\
\mathrm{LI}\end{array}$ & $\begin{array}{l}\text { SMI } \\
\text { SMI }\end{array}$ & $\begin{array}{l}\text { SLI } \\
\text { SMI }\end{array}$ & $\begin{array}{l}\text { EI } \\
\text { MI }\end{array}$ & $\begin{array}{l}\text { EI } \\
\text { EI }\end{array}$ & $\begin{array}{l}\text { SMI } \\
\text { SMI }\end{array}$ & $\begin{array}{l}\text { EI } \\
\text { EI }\end{array}$ & $\begin{array}{l}\text { SLI } \\
\text { SLL }\end{array}$ & $\begin{array}{l}\text { SLI } \\
\text { EI }\end{array}$ & $\begin{array}{l}\text { SMI } \\
\text { MI }\end{array}$ \\
\hline $\begin{array}{l}\text { 3.2.2 v c 3.3. } \\
\text { C.3.2v c C3.4 }\end{array}$ & $\begin{array}{l}\text { EI } \\
\text { EI } \\
E\end{array}$ & $\begin{array}{l}\text { EI } \\
\text { EI } \\
F \mid\end{array}$ & $\begin{array}{l}\text { EI } \\
\text { EI } \\
\text { E }\end{array}$ & $\begin{array}{l}\text { SLI } \\
\text { EI } \\
\text { El }\end{array}$ & $\begin{array}{l}\mathrm{MI} \\
\mathrm{MI}\end{array}$ & $\begin{array}{l}\text { 넌 } \\
\stackrel{4}{\longrightarrow}\end{array}$ & $\begin{array}{l}\text { MI } \\
\text { MI }\end{array}$ & $\begin{array}{l}\text { AMII } \\
\text { AMII } \\
\text { AMI }\end{array}$ & $\begin{array}{l}\text { SLI } \\
\text { SLI } \\
\text { SI }\end{array}$ & $\begin{array}{l}\text { SLI } \\
\text { EI }\end{array}$ & $\begin{array}{l}\mathrm{MI} \\
\mathrm{MI} \\
\mathrm{MI}\end{array}$ & $\begin{array}{l}\mathrm{MI} \\
\mathrm{MI}\end{array}$ & $\begin{array}{l}\text { SMI } \\
\text { MI }\end{array}$ & $\begin{array}{l}\text { SMI } \\
\text { SMI } \\
\text { SMI }\end{array}$ & $\begin{array}{l}\mathrm{MI} \\
\mathrm{MI}\end{array}$ & $\begin{array}{l}\text { SMI } \\
\text { SMI }\end{array}$ & $\begin{array}{l}\mathrm{EI} \\
\text { SMI }\end{array}$ & $\begin{array}{l}\text { EI } \\
\text { MII }\end{array}$ & $\begin{array}{l}\text { SLI } \\
\text { SLL }\end{array}$ & $\begin{array}{l}\mathrm{LI} \\
\mathrm{SLI}\end{array}$ & $\begin{array}{l}\text { EI } \\
\text { MI }\end{array}$ & $\begin{array}{l}\text { 는 } \\
\text { L }\end{array}$ & $\begin{array}{l}\text { LU } \\
\text { EI }\end{array}$ & $\begin{array}{l}E I \\
M I \\
M I I\end{array}$ \\
\hline $\begin{array}{l}\text { C3.2 v c } 3.5 .5 \\
\text { c3.2 v s C3.6. }\end{array}$ & $\begin{array}{l}\text { EI } \\
\text { EI } \\
\text { EI }\end{array}$ & $\begin{array}{l}\text { EI } \\
\text { EI } \\
\text { EI }\end{array}$ & $\begin{array}{l}\text { EI } \\
\text { SMI } \\
\text { EI }\end{array}$ & $\begin{array}{l}\text { EI } \\
\text { EI } \\
\text { EI }\end{array}$ & $\begin{array}{l}\mathrm{EI} \\
\mathrm{EI} \\
\mathrm{MI}\end{array}$ & $\begin{array}{l}\text { 녀 } \\
\text { L } \\
\end{array}$ & $\begin{array}{l}\text { SMI } \\
M I \\
M I\end{array}$ & $\begin{array}{l}\text { AMI } \\
\text { AMII } \\
\text { ALI }\end{array}$ & $\begin{array}{l}\text { SLI } \\
\text { SLI } \\
\text { SU }\end{array}$ & $\begin{array}{l}\text { EI } \\
\text { EI } \\
\text { E }\end{array}$ & $\begin{array}{l}\text { MI } \\
\text { MI } \\
\text { MI }\end{array}$ & $\begin{array}{l}\text { SII } \\
\text { SMI } \\
\text { SMI }\end{array}$ & $\begin{array}{l}\mathrm{MI} \\
\mathrm{MMI} \\
\mathrm{MI}\end{array}$ & $\begin{array}{l}\text { SMI } \\
\text { SMI } \\
\text { SMI }\end{array}$ & $\begin{array}{l}\mathrm{EI} \\
\mathrm{LI} \\
\mathrm{L}\end{array}$ & $\begin{array}{l}\text { SMI } \\
\text { SMI } \\
\text { SMI }\end{array}$ & $\begin{array}{l}\mathrm{EI} \\
\mathrm{EI} \\
\mathrm{EI}\end{array}$ & $\begin{array}{l}\text { SMII } \\
\text { MIMI } \\
\text { SWI }\end{array}$ & $\begin{array}{l}\text { AMI } \\
\text { SMI }\end{array}$ & $\begin{array}{l}\text { SMI } \\
\text { SMI }\end{array}$ & $\begin{array}{l}\mathrm{EI} \\
\mathrm{MI} \\
\mathrm{SI}\end{array}$ & $\begin{array}{l}\text { EII } \\
\text { SLI }\end{array}$ & $\begin{array}{l}\text { SLI } \\
\text { EI }\end{array}$ & $\begin{array}{l}\text { SMI } \\
\text { SMI }\end{array}$ \\
\hline 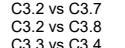 & $\begin{array}{l}\text { EI } \\
E I \\
E \\
E\end{array}$ & $\begin{array}{l}\text { EI } \\
\text { EI } \\
\text { EI }\end{array}$ & $\begin{array}{l}\text { SMI } \\
\text { SMI }\end{array}$ & $\begin{array}{l}\text { EI } \\
\text { EI } \\
\text { EI }\end{array}$ & $\begin{array}{l}\text { Mn } \\
\text { SMI } \\
\text { EI }\end{array}$ & $\begin{array}{l}\stackrel{\mathrm{L}}{\mathrm{L}} \\
\mathrm{M}\end{array}$ & $\begin{array}{l}\text { MII } \\
\text { SMI }\end{array}$ & $\begin{array}{l}\text { ALI } \\
\text { AMI } \\
\text { EI }\end{array}$ & $\begin{array}{l}\text { SLI } \\
\text { SLI } \\
\text { EI }\end{array}$ & $\begin{array}{l}\mathrm{EI} \\
\mathrm{EI}\end{array}$ & & $\begin{array}{l}\text { SMI } \\
\text { MI }\end{array}$ & $\begin{array}{l}\mathrm{MI} \\
\mathrm{SMI}\end{array}$ & $\begin{array}{l}\text { SMII } \\
\text { SMII }\end{array}$ & $\begin{array}{l}\mathrm{LI} \\
\mathrm{LI}\end{array}$ & $\begin{array}{l}\text { SMII } \\
\text { SMI }\end{array}$ & $\begin{array}{l}\text { EIII } \\
\text { SMI }\end{array}$ & $\begin{array}{l}\text { SMII } \\
\text { MIMI } \\
\text { SMI }\end{array}$ & $\begin{array}{l}\text { EI } \\
\text { EI }\end{array}$ & $\begin{array}{l}\text { SMI } \\
\text { SMII }\end{array}$ & $\begin{array}{l}\text { SMI } \\
\text { EI }\end{array}$ & $\begin{array}{l}\text { SLI } \\
\text { EI }\end{array}$ & $\begin{array}{l}\mathrm{EI} \\
\mathrm{SMI}\end{array}$ & $\begin{array}{l}\text { EIII } \\
\text { SMI }\end{array}$ \\
\hline 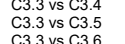 & $\begin{array}{l}\text { EI } \\
\text { EI } \\
\text { EI }\end{array}$ & $\begin{array}{l}\mathrm{E} \\
\text { SLI } \\
\text { EI }\end{array}$ & $\begin{array}{l}\text { MI } \\
\text { SMI } \\
\text { MI }\end{array}$ & $\begin{array}{l}\text { EI } \\
\text { EI } \\
\text { SLI }\end{array}$ & $\begin{array}{l}\mathrm{EI} \\
\mathrm{LI} \\
\mathrm{L}\end{array}$ & $\begin{array}{l}\text { MI } \\
\text { SMI } \\
\text { MMI }\end{array}$ & $\begin{array}{l}\mathrm{Ll} \\
\text { LL } \\
\text { SLI }\end{array}$ & $\begin{array}{l}\text { EII } \\
\text { AMI } \\
\text { AMI }\end{array}$ & & $\mathrm{E}$ & $\begin{array}{l}\text { EI } \\
\text { EI } \\
\text { EI }\end{array}$ & $\begin{array}{l}\text { MMI } \\
\text { MI } \\
\text { MI }\end{array}$ & $\begin{array}{l}\mathrm{LI} \\
\mathrm{EI} \\
\mathrm{MI}\end{array}$ & $\begin{array}{l}\text { SMI } \\
\text { SMI } \\
\text { SMII }\end{array}$ & $\begin{array}{l}\text { MLI } \\
\text { MLI }\end{array}$ & $\begin{array}{l}\text { SLI } \\
\text { SLI }\end{array}$ & $\begin{array}{l}\text { EI } \\
\text { SLI } \\
\text { SU }\end{array}$ & $\begin{array}{l}\text { SMII } \\
E \mid \\
E \mid\end{array}$ & $\begin{array}{l}\text { LI } \\
\text { SLI }\end{array}$ & $\begin{array}{l}E I \\
E I \\
\text { EI }\end{array}$ & $\begin{array}{l}E I \\
E I \\
E I\end{array}$ & $\begin{array}{l}E I \\
\text { EI } \\
|l| l\end{array}$ & $\begin{array}{l}\text { MI } \\
\text { SMI }\end{array}$ & $\begin{array}{l}M I \\
\text { EI }\end{array}$ \\
\hline 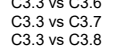 & EI & $\begin{array}{l}\text { EI } \\
\text { EII } \\
\text { EI }\end{array}$ & $\begin{array}{l}\text { EI } \\
\text { SMI }\end{array}$ & $\begin{array}{l}\text { EI } \\
\mathrm{EI}\end{array}$ & EI & $\begin{array}{l}\text { MMI } \\
\text { AMI } \\
\text { MI }\end{array}$ & $\begin{array}{l}\text { SLI } \\
\text { SLI }\end{array}$ & $\begin{array}{l}\text { EMI } \\
\text { EI } \\
\text { EI }\end{array}$ & & $\begin{array}{l}\text { EI } \\
\text { EI } \\
\text { EI }\end{array}$ & MMI & $\begin{array}{l}\text { MI } \\
\text { EI } \\
\text { EI }\end{array}$ & $\begin{array}{l}\text { MI } \\
\text { EI } \\
\text { EI }\end{array}$ & $\begin{array}{l}\text { SMI } \\
\text { EII } \\
E !\end{array}$ & $\begin{array}{l}\mathrm{ALI} \\
\mathrm{LI} \\
\mathrm{MLI}\end{array}$ & $\begin{array}{l}\text { EI } \\
\text { EI } \\
\text { | }\end{array}$ & $\begin{array}{l}\text { SLI } \\
\text { SLI }\end{array}$ & $\begin{array}{l}\text { EI } \\
\text { EI } \\
E\end{array}$ & $\begin{array}{l}\text { SLI } \\
\text { SLI }\end{array}$ & $\begin{array}{l}\text { SMI } \\
\text { SMI }\end{array}$ & MI & $\begin{array}{l}\text { MI } \\
M I\end{array}$ & $\begin{array}{l}\text { SMI } \\
\text { EI }\end{array}$ & $\begin{array}{l}E I \\
\text { EI } \\
\text { EI }\end{array}$ \\
\hline 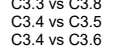 & $\begin{array}{l}\text { EI } \\
\text { EI } \\
\text { EI }\end{array}$ & $\begin{array}{l}\text { EI } \\
\text { EI } \\
\text { SLI }\end{array}$ & $\begin{array}{l}\text { SII } \\
\text { SII }\end{array}$ & $\begin{array}{l}\text { SMI } \\
\text { SMI }\end{array}$ & 崖 & $\begin{array}{l}\text { AMI } \\
\mathrm{EI}\end{array}$ & $\begin{array}{l}\text { SLI } \\
\text { MI }\end{array}$ & $\begin{array}{l}\text { MLI } \\
\text { AMI }\end{array}$ & EI & $\begin{array}{ll}E & E \\
\text { EI } \\
\text { EI }\end{array}$ & MII & $\begin{array}{l}\text { EI } \\
\text { EI } \\
\text { SL }\end{array}$ & $\begin{array}{l}\text { EI } \\
\text { EI } \\
\text { MI }\end{array}$ & 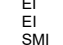 & $\begin{array}{l}\text { MLI } \\
\longleftrightarrow\end{array}$ & $\begin{array}{l}\mathrm{EI} \\
\mathrm{EI} \\
\mathrm{EI}\end{array}$ & $\begin{array}{l}M I \\
\text { EI } \\
\text { SMI }\end{array}$ & $\begin{array}{l}\text { M1 } \\
\text { sil }\end{array}$ & EI & $\begin{array}{l}\text { SMI } \\
\text { EI }\end{array}$ & $\begin{array}{ll}E I \\
\text { LI }\end{array}$ & $\begin{array}{l}\text { MI } \\
\text { EI } \\
S \text { s. }\end{array}$ & \begin{tabular}{l} 
MI \\
\multicolumn{1}{|c|}{}
\end{tabular} & $\begin{array}{l}\text { SII } \\
\mathrm{El}\end{array}$ \\
\hline 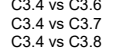 & $\begin{array}{l}\text { EI } \\
\text { EI } \\
\text { EI }\end{array}$ & $\begin{array}{l}\text { SLI } \\
\text { EI } \\
\text { EI }\end{array}$ & $\begin{array}{l}\mathrm{LI} \\
\mathrm{SLI}\end{array}$ & $\begin{array}{l}\text { EI } \\
\text { SMI }\end{array}$ & $\begin{array}{l}\text { EI } \\
\text { EI } \\
\text { EI }\end{array}$ & $\begin{array}{l}\mathrm{M} \\
\text { SMI }\end{array}$ & $\begin{array}{l}\text { MI } \\
\text { SLI }\end{array}$ & & 政 & E & $\begin{array}{l}\text { SLI } \\
\text { SMMI }\end{array}$ & $\begin{array}{l}\text { SLI } \\
\text { SMI } \\
\text { SMI }\end{array}$ & $\begin{array}{l}\text { MI } \\
E I \\
E \in \mid\end{array}$ & 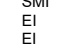 & $\begin{array}{l}\text { SMI } \\
\text { SMI }\end{array}$ & $\begin{array}{l}\text { SMI } \\
\text { SMI } \\
\text { EI }\end{array}$ & $\begin{array}{l}\text { SMI } \\
\text { SII }\end{array}$ & $\begin{array}{l}\text { SLI } \\
\text { SLI } \\
\text { EI }\end{array}$ & SMI & $\begin{array}{l}\text { SLI } \\
\text { SMI }\end{array}$ & SMI & $\begin{array}{l}\text { SMI } \\
\text { EI }\end{array}$ & $\begin{array}{l}\text { SLI } \\
\text { SLI }\end{array}$ & $\begin{array}{l}\text { SLI } \\
\text { SLI }\end{array}$ \\
\hline $\begin{array}{l}\text { C3.5 vs } 3.6 \\
\text { C3.5 v } C 3.7\end{array}$ & MI & $\begin{array}{l}\text { EI } \\
\text { EI } \\
\text { EI }\end{array}$ & $\begin{array}{lll}\mathrm{E} \\
\mathrm{E} \\
\mathrm{E}\end{array}$ & $\begin{array}{l}\text { EI } \\
\text { EI } \\
\text { El }\end{array}$ & EI & $\stackrel{M}{M I I}$ & $\begin{array}{l}\mathrm{MI} \\
\text { SMI }\end{array}$ & & & EI & $\begin{array}{l}\text { MMII } \\
\text { MMI }\end{array}$ & $\begin{array}{l}\text { SMI } \\
\text { SMI } \\
\mathrm{EI}\end{array}$ & MI & $\begin{array}{l}\mathrm{L} \\
\text { SMI } \\
\text { El }\end{array}$ & MMI & $\begin{array}{l}\text { EI } \\
\text { EI } \\
\text { SIM }\end{array}$ & $\begin{array}{l}\mathrm{sin} \\
\mathrm{SSI}\end{array}$ & $\begin{array}{l}\text { le } \\
\text { EI } \\
\text { EI }\end{array}$ & $\begin{array}{l}\text { SIII } \\
\text { EI }\end{array}$ & $\begin{array}{l}E I \\
\text { EI } \\
\text { SIM }\end{array}$ & MII & MMI & SMI & $\begin{array}{l}\text { SWI } \\
\text { EI }\end{array}$ \\
\hline 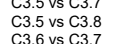 & EI & $\begin{array}{l}E I \\
\text { SMI } \\
\text { EI }\end{array}$ & $\begin{array}{l}\text { II } \\
\text { SLI }\end{array}$ & $\begin{array}{l}\text { SSMI } \\
\text { EI }\end{array}$ & 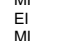 & EI & $\begin{array}{l}\text { SLI } \\
\text { LI }\end{array}$ & $\begin{array}{ll}E l \\
\text { AMI }\end{array}$ & 是 & $\begin{array}{l}\text { EI } \\
\text { EI } \\
\text { EI }\end{array}$ & 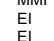 & $\begin{array}{l}E I \\
E I \\
E I \\
E I\end{array}$ & $\begin{array}{l}\text { EI } \\
\text { EI }\end{array}$ & $\begin{array}{l}E I \\
\text { EI } \\
\text { SU }\end{array}$ & $\begin{array}{l}\text { SMII } \\
\text { SMMI }\end{array}$ & $\begin{array}{l}\text { SMI } \\
\text { SMI } \\
\text { M }\end{array}$ & $\begin{array}{l}\text { SII } \\
\text { SMI } \\
\text { FI }\end{array}$ & MI & $\begin{array}{l}\text { SMI } \\
\text { SMI }\end{array}$ & $\begin{array}{l}\text { SMI } \\
\text { EI } \\
\text { s.m. }\end{array}$ & EI & $\begin{array}{l}\text { ELI } \\
\text { SLI }\end{array}$ & $\begin{array}{l}\text { SLI } \\
\text { SMI }\end{array}$ & $\begin{array}{l}\text { SII } \\
\text { MI }\end{array}$ \\
\hline 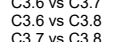 & $\begin{array}{l}\text { EI } \\
\text { EI } \\
\text { EI }\end{array}$ & $\begin{array}{l}\mathrm{EI} \\
\text { SMI } \\
\mathrm{EI}\end{array}$ & $\begin{array}{l}\text { SII } \\
\text { MI }\end{array}$ & $\begin{array}{l}\text { El } \\
\text { EI } \\
\text { EI }\end{array}$ & $\begin{array}{l}\text { MI } \\
\text { EI } \\
\text { EI }\end{array}$ & $\begin{array}{l}\text { L } \\
\text { L } \\
\text { L }\end{array}$ & & AMI & $\begin{array}{l}\text { EI } \\
\text { EI } \\
\text { EI }\end{array}$ & $\begin{array}{l}\text { EI } \\
\text { EI } \\
\text { EI }\end{array}$ & $\begin{array}{l}\mathrm{EI} \\
\text { SMI } \\
\mathrm{EI}\end{array}$ & $\begin{array}{l}\mathrm{EL} \\
\text { SLI } \\
\mathrm{EI}\end{array}$ & 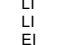 & $\begin{array}{l}\text { SLI } \\
\text { SLI } \\
\text { EI }\end{array}$ & $\begin{array}{l}\text { MMI } \\
\text { SLI } \\
\text { SLI }\end{array}$ & $\begin{array}{l}\text { EI } \\
\text { SLI } \\
\text { EI }\end{array}$ & $\begin{array}{l}\text { EI } \\
\text { SMI } \\
\text { SMI }\end{array}$ & $\begin{array}{l}E I \\
\text { MI } \\
M 1\end{array}$ & $\begin{array}{l}\mathrm{EI} \\
\text { SMI } \\
\mathrm{EI}\end{array}$ & $\begin{array}{l}\text { SMI } \\
\text { SMI }\end{array}$ & 酐 & $\begin{array}{ll}L 1 \\
4 \\
s\end{array}$ & $\begin{array}{l}\text { SLI } \\
\text { SLI }\end{array}$ & SMI \\
\hline $\begin{array}{l}\text { C4. v v s } 4.2 \\
\text { C.1. v } 54.3\end{array}$ & $\begin{array}{l}\text { EI } \\
\text { EI } \\
\text { EI }\end{array}$ & $\begin{array}{l}\mathrm{EI} \\
\text { EI } \\
\text { SMI }\end{array}$ & $\begin{array}{l}\text { MII } \\
\text { MMI } \\
\text { MMI }\end{array}$ & $\begin{array}{l}\text { EI } \\
\text { EI } \\
\text { SMI }\end{array}$ & $\begin{array}{l}\text { EI } \\
\text { EI } \\
\text { EI }\end{array}$ & MI & & EII & $\begin{array}{l}\text { SMI } \\
\text { SMI }\end{array}$ & $\begin{array}{l}\mathrm{EI} \\
\mathrm{MI} \\
\mathrm{SMI}\end{array}$ & $\begin{array}{l}E I \\
\text { EI } \\
\text { EI }\end{array}$ & $\begin{array}{l}E I \\
\text { EI } \\
\text { SMI }\end{array}$ & $\begin{array}{l}\mathrm{EI} \\
\mathrm{SLI} \\
\mathrm{EI}\end{array}$ & $\begin{array}{l}\text { SII } \\
\text { MI }\end{array}$ & $\begin{array}{l}\text { SLI } \\
\text { MMI }\end{array}$ & $\begin{array}{l}\text { EI } \\
\text { SMI } \\
\text { SMI }\end{array}$ & $\begin{array}{l}\text { SII } \\
\text { MI }\end{array}$ & $\begin{array}{l}\mathrm{MI} \\
\mathrm{MI} \\
\mathrm{MI}\end{array}$ & $\begin{array}{l}E I \\
\text { EI } \\
\text { EI }\end{array}$ & MI & $\begin{array}{l}\text { EI } \\
\text { EI } \\
\text { MI }\end{array}$ & AMI & $\begin{array}{l}\text { SMI } \\
\text { EI }\end{array}$ & $\begin{array}{l}\text { SLI } \\
\text { SLI }\end{array}$ \\
\hline 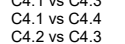 & $\begin{array}{ll} & \text { EI } \\
\text { EI } \\
\text { EI }\end{array}$ & $\begin{array}{l}\text { SMI } \\
\text { MI }\end{array}$ & $\begin{array}{l}\text { MIII } \\
\text { MI }\end{array}$ & $\begin{array}{l}\text { SMII } \\
\text { SMII }\end{array}$ & EI & & & $\begin{array}{l}\text { AAll } \\
\text { All } \\
E 1\end{array}$ & $\begin{array}{l}\text { SMI } \\
\text { SII }\end{array}$ & $\begin{array}{l}\text { SMI } \\
\text { SLI }\end{array}$ & $\begin{array}{l}\mathrm{EI} \\
\mathrm{MI}\end{array}$ & $\begin{array}{l}\text { MIII } \\
\text { SMI }\end{array}$ & $\begin{array}{l}\text { EI } \\
\text { EI }\end{array}$ & $\begin{array}{l}\text { SLI } \\
\text { MI }\end{array}$ & $\begin{array}{l}\text { MMI } \\
\text { AMI }\end{array}$ & $\begin{array}{l}\text { SMI } \\
\mathrm{EI}\end{array}$ & $\begin{array}{l}\text { MWI } \\
\text { EI } \\
\text { EI }\end{array}$ & $\begin{array}{l}\text { MII } \\
\text { EI } \\
\text { EI }\end{array}$ & $\begin{array}{ll} & \text { EI } \\
\text { EI } \\
\text { EI }\end{array}$ & $\begin{array}{l}\text { SII } \\
\text { EII }\end{array}$ & $\begin{array}{l}\text { MI } \\
\text { EI } \\
\text { MI }\end{array}$ & $\begin{array}{l}\mathrm{EI} \\
\mathrm{SMI} \\
\mathrm{LI}\end{array}$ & $\begin{array}{l}\text { NI } \\
\text { SMI } \\
\text { SMI }\end{array}$ & $\begin{array}{l}\text { SII } \\
\text { MI }\end{array}$ \\
\hline $\begin{array}{l}\text { C4.2 v s C.4. } \\
\text { C.4.3 vs C4.4 }\end{array}$ & EI & $\begin{array}{l}\text { MII } \\
\text { MI }\end{array}$ & $\begin{array}{l}\mathrm{MI} \\
\mathrm{EI}\end{array}$ & 焉 & EI & SMI & $\begin{array}{l}\text { MLI } \\
\text { SLI }\end{array}$ & $\begin{array}{l}\text { EI } \\
\text { EP }\end{array}$ & $\begin{array}{l}\mathrm{E} 1 \mathrm{SI} \\
\mathrm{EI}\end{array}$ & $\begin{array}{l}\mathrm{El} \\
\mathrm{EI}\end{array}$ & $\begin{array}{l}\mathrm{MI} \\
\mathrm{MI}\end{array}$ & $\begin{array}{l}\text { SMII } \\
\text { SLI }\end{array}$ & $\begin{array}{l}\mathrm{MI} \\
\mathrm{EI}\end{array}$ & $\begin{array}{l}M \\
M \\
M I \\
L I\end{array}$ & $\begin{array}{l}\text { AMI } \\
\text { MMI }\end{array}$ & $\begin{array}{l}\mathrm{El} \\
\mathrm{EI} \\
\mathrm{EI}\end{array}$ & $\begin{array}{l}\mathrm{El} \\
\mathrm{EI} \\
\mathrm{EI}\end{array}$ & $\begin{array}{ll}\text { SLI } \\
\text { EI }\end{array}$ & $\begin{array}{l}\mathrm{EI} \\
\mathrm{EI} \\
\mathrm{EI}\end{array}$ & $\begin{array}{l}\text { SLI } \\
\text { SLI }\end{array}$ & $\begin{array}{l}\text { EI } \\
\text { MLI }\end{array}$ & $\begin{array}{l}\text { MLI } \\
\text { EI }\end{array}$ & $\begin{array}{l}\mathrm{SI} \\
\mathrm{El} \\
\mathrm{LI}\end{array}$ & $\begin{array}{l}\text { MII } \\
\text { SLI }\end{array}$ \\
\hline 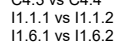 & $\begin{array}{l}\text { EI } \\
\text { EI } \\
\text { EI }\end{array}$ & 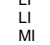 & $\begin{array}{l}\mathrm{LI} \\
\mathrm{L} \\
\mathrm{MI}\end{array}$ & $\begin{array}{l}\text { EI } \\
\text { SLI }\end{array}$ & EI & & & $\begin{array}{l}\mathrm{EL} \\
\text { SLI } \\
\mathrm{EI}\end{array}$ & $\begin{array}{l}\mathrm{EI} \\
\mathrm{EI} \\
\mathrm{SLI}\end{array}$ & $\begin{array}{l}\text { SLI } \\
\text { MLI }\end{array}$ & $\begin{array}{l}\text { SMI } \\
\text { SMI }\end{array}$ & $\begin{array}{l}\text { MLI } \\
\text { MLI }\end{array}$ & $\begin{array}{l}\text { EI } \\
\text { SMI }\end{array}$ & $\begin{array}{l}\text { EI } \\
\text { EI }\end{array}$ & $\begin{array}{l}\mathrm{LI} \\
\mathrm{EI}\end{array}$ & $\begin{array}{l}\mathrm{LI} \\
\mathrm{EI} \\
\mathrm{El}\end{array}$ & $\begin{array}{ll}\text { EI } \\
\text { EI } \\
\text { EI }\end{array}$ & II & $\begin{array}{l}\mathrm{El} \\
\mathrm{EI} \\
\mathrm{SLI}\end{array}$ & $\begin{array}{l}\text { EI } \\
\text { SLI }\end{array}$ & $\begin{array}{l}\text { ALI } \\
\text { ALI }\end{array}$ & $\begin{array}{l}\mathrm{MI} \\
\text { SMI }\end{array}$ & $\begin{array}{l}\mathrm{L} \\
\mathrm{E} \\
\mathrm{MI}\end{array}$ & $\begin{array}{l}\text { SLI } \\
\text { SLI }\end{array}$ \\
\hline 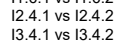 & $\begin{array}{l}\text { EI } \\
\text { SLI }\end{array}$ & $\begin{array}{l}\text { SMI } \\
\mathrm{EI}\end{array}$ & $\begin{array}{l}\text { MMI } \\
\text { MLI }\end{array}$ & $\begin{array}{l}\text { SMI } \\
\text { SLI }\end{array}$ & & & & $\begin{array}{l}\text { MMI } \\
\mathrm{EI}\end{array}$ & $\begin{array}{ll}\mathrm{MI} \\
\mathrm{LI}\end{array}$ & $\begin{array}{l}\mathrm{El} \\
\mathrm{El}\end{array}$ & $\begin{array}{l}\text { MI } \\
\text { SMI }\end{array}$ & $\begin{array}{l}\mathrm{EI} \\
\mathrm{EL}\end{array}$ & $\begin{array}{l}\text { MMI } \\
\text { MLI }\end{array}$ & $\begin{array}{l}\text { SMI } \\
L I\end{array}$ & $\begin{array}{l}\mathrm{EI} \\
\mathrm{MLI}\end{array}$ & $\begin{array}{l}\text { SMI } \\
\mathrm{LI}\end{array}$ & SLI & $\begin{array}{l}\mathrm{SMI} \\
\mathrm{LI}\end{array}$ & $\begin{array}{l}\mathrm{MI} \\
\mathrm{SMI}\end{array}$ & $\begin{array}{l}\text { SLI } \\
\text { SMI }\end{array}$ & $\begin{array}{l}\text { EI } \\
\text { El }\end{array}$ & $\begin{array}{l}\text { MMI } \\
\mathrm{LI}\end{array}$ & $\begin{array}{l}\mathrm{Ml} \\
\mathrm{LI}\end{array}$ & $\begin{array}{l}\mathrm{MI} \\
\mathrm{MI}\end{array}$ \\
\hline 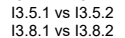 & SLI & $\begin{array}{l}\mathrm{MI} \\
\mathrm{MMI}\end{array}$ & $\begin{array}{l}\text { AMI } \\
\text { MLI }\end{array}$ & $\begin{array}{l}\text { SLI } \\
\text { SLI }\end{array}$ & & & & $\begin{array}{c}\bar{M} \mid \\
\mathrm{EI}\end{array}$ & $\begin{array}{l}\mathrm{MI} \\
\mathrm{MI}\end{array}$ & MI & $\underset{E I}{M I}$ & EI & $\begin{array}{l}\mathrm{LL} \\
\mathrm{MLI}\end{array}$ & $\begin{array}{l}\text { MLI } \\
E I\end{array}$ & $\begin{array}{l}\text { SLI } \\
\text { MI }\end{array}$ & $\begin{array}{l}\mathrm{EI} \\
\mathrm{MLI}\end{array}$ & $\begin{array}{l}\mathrm{MII} \\
\mathrm{MI}\end{array}$ & MLI & $\begin{array}{l}\text { SLI } \\
\text { LI }\end{array}$ & $\begin{array}{l}\text { SLI } \\
\text { SMI }\end{array}$ & EI & $\begin{array}{l}\text { MLI } \\
\text { SLI }\end{array}$ & $\underset{E I}{\text { MMI }}$ & $\underset{M I I}{\mathrm{MI}}$ \\
\hline
\end{tabular}

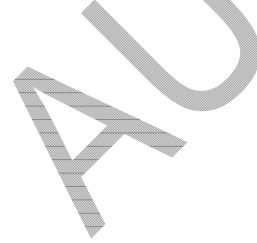




\subsection{Weighting of the elements in the decision-making tree}

The weighting of the elements in the hierarchy into which SIRSDEC is structured is essential to support its realistic application. The AHP method (Saaty, 1990) was used to assess the pairwise comparisons of expert judgments. Table 4 includes the values of C.R. obtained after evaluating the consistency of the comparisons received from each expert in relation to requirements and criteria. The results showed that the number of inconsistent comparisons exceeded $50 \%$ for each group (C.R. > 0.1). The comparisons associated with the economic and environmental criteria included 13 inconsistencies each, whilst those related to social criteria and the four requirements involved 14 and 12 inconsistencies, respectively. The highest number of inconsistencies (16) corresponded to the management criteria.

Table 4. Summary of the consistency analysis of the comparisons provided by the respondents

\begin{tabular}{lccccc}
\hline \multirow{2}{*}{ Respondent \# } & Requirement & $\begin{array}{c}\text { Economic } \\
\text { criteria }\end{array}$ & $\begin{array}{c}\text { Social } \\
\text { criteria }\end{array}$ & $\begin{array}{c}\text { Management } \\
\text { criteria }\end{array}$ & $\begin{array}{c}\text { Environmental } \\
\text { criteria }\end{array}$ \\
\cline { 2 - 6 } & C.R. & C.R. & C.R. & C.R. & C.R. \\
\hline 1 & 0.000 & 0.000 & 0.237 & 0.000 & 0.028 \\
2 & 0.139 & 0.150 & 0.076 & 0.087 & 0.047 \\
3 & 0.166 & 0.178 & 0.255 & 0.193 & 0.208 \\
4 & 0.058 & 0.058 & 0.080 & 0.068 & 0.090 \\
5 & 0.000 & 0.131 & 0.123 & 0.092 & 0.041 \\
6 & 0.292 & 0.124 & 0.165 & 0.090 & 0.308 \\
7 & 0.171 & 0.210 & 0.404 & 0.235 & 0.182 \\
8 & 0.644 & 0.257 & 0.376 & 0.734 & 1.391 \\
9 & 0.000 & 0.059 & 0.076 & 0.218 & 0.003 \\
10 & 0.184 & 0.043 & 0.139 & 0.088 & 0.012 \\
11 & 0.131 & 0.132 & 0.250 & 0.373 & 0.278 \\
12 & 0.181 & 0.105 & 0.274 & 0.442 & 0.332 \\
13 & 0.098 & 0.012 & 0.262 & 0.231 & 0.124 \\
14 & 0.059 & 0.551 & 0.046 & 0.153 & 0.041 \\
15 & 0.086 & 0.226 & 0.281 & 0.204 & 0.315 \\
16 & 0.120 & 0.000 & 0.034 & 0.045 & 0.093 \\
17 & 0.012 & 0.127 & 0.420 & 0.130 & 0.122 \\
18 & 0.000 & 0.103 & 0.070 & 0.135 & 0.051 \\
19 & 0.000 & 0.000 & 0.035 & 0.126 & 0.252 \\
20 & 0.184 & 0.016 & 0.086 & 0.193 & 0.213 \\
21 & 0.197 & 0.005 & 0.105 & 0.079 & 0.082 \\
22 & 0.201 & 0.057 & 0.126 & 0.373 & 0.371 \\
23 & 0.000 & 0.005 & 0.073 & 0.254 & 0.171 \\
24 & 0.000 & 0.169 & 0.098 & 0.355 & 0.088 \\
\hline Inconsistent comparisons & 12 & 11 & 10 & 8 & 11 \\
& & 13 & 14 & 16 & 13 \\
\hline
\end{tabular}

The Grubbs' test (Grubbs, 1950) was undertaken to detect outliers in the values of C.R. (see Figure 2). Respondent \#8 provided extreme comparisons in relation to requirements, management criteria and environmental criteria, whilst respondent \#14 was too inconsistent with respect to the economic criteria. 


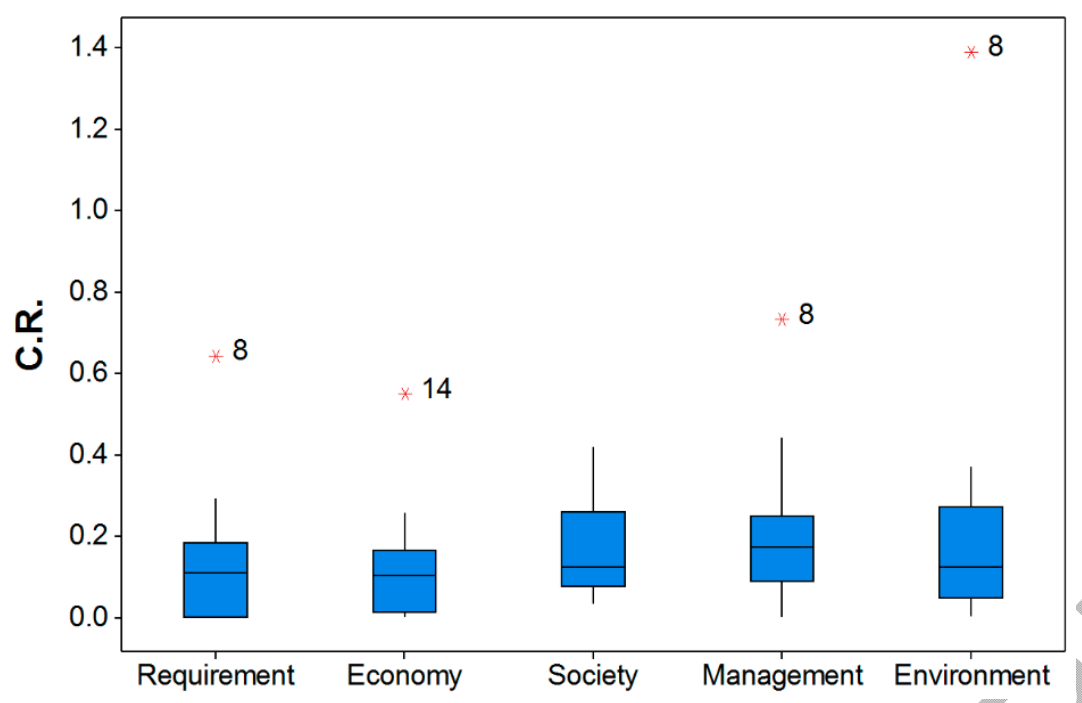

Figure 2. Boxplots of the values of C.R. associated with the pairwise comparisons provided by the experts

The methodology proposed by Jato-Espino et al. (2016), consisting on the Generalized Reduced Gradient (GRG) algorithm (Abadie et al., 1968) and an aggregation system based on the proximity between the judgments of each pair of respondents, was used to adjust the inconsistent judgments found in the returned questionnaires and integrate them into a consensual set of weights. The application of the GRG algorithm made consistent every comparison with a value of C.R. > 0.1, except those provided by the respondent \#8, who proved to be too inconsistent with respect to several comparisons and was therefore discarded for further analyses. Since the remaining experts were found to be too inconsistent only for one isolated comparison each at most, their remaining judgments were considered henceforth.

Table 5 shows the consensual weights obtained for each element of the decision-making tree after aggregating the consistent judgments of the experts according to their similarity of thought, in order to give more importance to those who proved to have closer points of view. The social dimension reached the highest weight (0.324), followed by Environment (0.289), Economy (0.247) and Management (0.140). These values ensured the achievement of a balance among the weights of the pillars of sustainable development. Combating poverty (C4.1) was found to be the most important factor in economic terms, whilst Natural Ecosystems conservation (C3.1) and Biodiversity Ecosystem (C3.2) were the criteria with the highest weights in the environmental domain. As for the social requirement, three criteria highlighted over the rest: Role of indigenous people \& communities (C2.2), Equitable Development (C2.3) and Social impacts \& Benefits (C2.4). Finally, the results demonstrated that Project Sustainability Management plan (C1.2) was the most relevant criterion in the management category. 
Table 5. Weights for the elements in the SIRSDEC decision-making tree

\begin{tabular}{|c|c|c|c|c|c|}
\hline $\begin{array}{c}\text { Requirement } \\
\text { R\# }\end{array}$ & $\begin{array}{c}\text { Weight } \\
\mathrm{W}_{\mathrm{R} \#}\end{array}$ & $\begin{array}{c}\text { Criteria } \\
\text { C\#.\# }\end{array}$ & $\begin{array}{c}\text { Weight } \\
\mathbf{W}_{\text {C\#.\#\# }}\end{array}$ & $\begin{array}{l}\text { Indicator } \\
\text { I\#.\#.\# }\end{array}$ & $\begin{array}{c}\text { Weight } \\
\text { W I\#.\#.\# }^{\text {Int }}\end{array}$ \\
\hline \multirow{8}{*}{ R1 } & \multirow{8}{*}{0.140} & \multirow{2}{*}{ C1.1 } & \multirow{2}{*}{0.112} & I1.1.1 & 1.000 \\
\hline & & & & |1.1.2 & 1.000 \\
\hline & & C1.2 & 0.230 & $\mid 1.2 .1$ & 1.000 \\
\hline & & C1.3 & 0.148 & |1.3.1 & 1.000 \\
\hline & & C1.4 & 0.135 & |1.4.1 & 1.000 \\
\hline & & C1.5 & 0.171 & |1.5.1 & 1.000 \\
\hline & & \multirow{2}{*}{ C1.6 } & \multirow{2}{*}{0.204} & |1.6.1 & 1.000 \\
\hline & & & & |1.6.2 & 1.000 \\
\hline \multirow{6}{*}{$\mathrm{R} 2$} & \multirow{6}{*}{0.324} & C2.1 & 0.179 & I2.1.1 & 1.000 \\
\hline & & $\mathrm{C} 2.2$ & 0.214 & I2.2.1 & 1.000 \\
\hline & & C2.3 & 0.211 & I2.3.1 & 1.000 \\
\hline & & \multirow{2}{*}{$\mathrm{C} 2.4$} & \multirow{2}{*}{0.222} & |2.4.1 & 1.000 \\
\hline & & & & $\mid 2.4 .2$ & 1.000 \\
\hline & & $\mathrm{C} 2.5$ & 0.174 & I2.5.1 & 1.000 \\
\hline \multirow{11}{*}{ R3 } & \multirow{11}{*}{0.289} & C3.1 & 0.169 & I3.1.1 & 1.000 \\
\hline & & C3.2 & 0.155 & |3.2.1 & 1.000 \\
\hline & & C3.3 & 0.130 & |3.3.1 & 1.000 \\
\hline & & \multirow{2}{*}{ C3.4 } & \multirow{2}{*}{0.102} & I3.4.1 & 1.000 \\
\hline & & & & 13.4 .2 & 1.000 \\
\hline & & \multirow{2}{*}{ C3.5 } & \multirow{2}{*}{0.143} & |3.5.1 & 1.000 \\
\hline & & & & I3.5.2 & 1.000 \\
\hline & & C3.6 & 0.094 & |3.6.1 & 1.000 \\
\hline & & C3.7 & 0.109 & 13.7.1 & 1.000 \\
\hline & & \multirow{2}{*}{ C3.8 } & 0.098 & I3.8.1 & 1.000 \\
\hline & & & 0.000 & I3.8.2 & 1.000 \\
\hline \multirow{4}{*}{ R4 } & \multirow{4}{*}{0.247} & C4.1 & 0.398 & 14.1 .1 & 1.000 \\
\hline & & C4.2 & 0.256 & |4.2.1 & 1.000 \\
\hline & & C4.3 & 0.145 & |4.3.1 & 1.000 \\
\hline & & C4.4 & 0.201 & |4.4.1 & 1.000 \\
\hline
\end{tabular}

156

157

158

159

160

161

162

163

164

165

166

167

168

169

170

171

172

173

174

175

176

177

178

179

\subsection{Characterization of indicators using value functions}

The eight indicators included in the management requirement promote the use of effective project governance frameworks, sustainable best practices and standards focused on enhancing management in infrastructure projects. Binary stepped value functions were assigned to all indicators in this category. Hence, 0 or 1 points are allocated to them depending on whether the goals they seek are met or not. The same principle was also applied to indicators I2.5.1 and 14.1.1. Regarding the social requirement, indicators 12.1 .1 and 12.2 .1 are also rated using binary stepped functions according to standards of the International Association for Public Participation (IAP2, 2016). Projects in which stakeholders, population and/or indigenous community are at least involved are rewarded with 1 point, otherwise they are rated with 0 points.

Increasing linear functions were set for indicators I3.3.1, 13.4.1, 13.4.2, 13.5.1, 13.5.2, 13.8.1 and I3.8.2 to reward the performance of indicators proportionally. Due to the scarcity of metrics for developing countries, the lower and upper values for these value functions were based on thresholds established by existing sustainable infrastructure rating systems (Envision (ISI, 2012), Civil Engineering Environmental Quality (CEEQUAL, 2015) and Infrastructure Sustainability (IS) Rating Tool (ISCA, 2012)) for equivalent indicators. The minimum and maximum values for indicators I2.4.2, I3.1.1, 13.2.1 and 14.2.1 were extracted from the same data source. These indicators were characterized through concave value functions, in order to reward projects that have low values with respect to them. Indicators I2.3.1 and I4.1.1 were represented by increasing convex value functions, with their bounds delimited according to reports from the International Labor Organization (ILO) (ILO, 2015).

S-shape was found to be the most appropriate value function for indicators I2.4.1, I3.6.1, I3.7.1 and 14.3.1. These indicators were defined again from thresholds found in existing infrastructure rating systems, with the exception of 13.7.1, 
whose range of values was taken from the NEC directive 2001/81/EC (EU, 2016). Table 6 summarizes the parameters that characterize the value functions defined for each indicator included in SIRSDEC.

Table 6. Parameters established for the value functions to characterize each indicator in SIRSDEC

\begin{tabular}{|c|c|c|c|c|c|c|}
\hline Indicator & Xmin & Xmax & $\mathbf{P i}$ & $\mathrm{Ci}$ & $\mathbf{K i}$ & Function \\
\hline | 1.1.1 & 0.00 & 1.00 & n.a. & n.a. & n.a. & Stepped \\
\hline | 1.1 .2 & 0.00 & 1.00 & n.a. & n.a. & n.a. & Stepped \\
\hline | 1.2.1 & 0.00 & 1.00 & n.a. & n.a. & n.a. & Stepped \\
\hline | 1.3.1 & 0.00 & 1.00 & n.a. & n.a. & n.a. & Stepped \\
\hline | 1.4.1 & 0.00 & 1.00 & n.a. & n.a. & n.a. & Stepped \\
\hline | 1.5.1 & 0.00 & 1.00 & n.a. & n.a. & n.a. & Stepped \\
\hline | 1.6.1 & 0.00 & 1.00 & n.a. & n.a. & n.a. & Stepped \\
\hline I 1.6.2 & 0.00 & 1.00 & n.a. & n.a. & n.a. & Stepped \\
\hline I 2.1.1 & 0.00 & 1.00 & n.a. & n.a. & n.a. & Stepped \\
\hline | 2.2.1 & 0.00 & 1.00 & n.a. & n.a. & n.a. & Stepped \\
\hline | 2.3.1 & 51.00 & 99.00 & 0.75 & 51.00 & 4.000 & Convex \\
\hline | 2.4 .1 & 25.00 & 0.00 & 3.00 & 5.00 & 0.020 & S-Shape \\
\hline I 2.4 .2 & 20.00 & 0.00 & 5.00 & 3.00 & 0.002 & Concave \\
\hline | 2.5.1 & 0.00 & 1.00 & n.a. & n.a. & n.a. & Stepped \\
\hline I 3.1.1 & 30.00 & 0.00 & 2.50 & 15.00 & 0.050 & Concave \\
\hline | 3.2.1 & 30.00 & 0.00 & 2.50 & 15.00 & 0.050 & Concave \\
\hline | 3.3.1 & 10.00 & 40.00 & 1.00 & 40.00 & 1.000 & Linear \\
\hline | 3.4.1 & 10.00 & 30.00 & 1.00 & 30.00 & 1.000 & Linear \\
\hline | 3.4 .2 & 10.00 & 25.00 & 1.00 & 25.00 & 1.000 & Linear \\
\hline | 3.5.1 & 5.00 & 20.00 & 1.00 & 20.00 & 1.000 & Linear \\
\hline | 3.5.2 & 0.00 & 30.00 & 1.00 & 30.00 & 1.000 & Linear \\
\hline | 3.6.1 & 15.00 & 0.00 & 5.00 & 8.00 & 0.250 & S-Shape \\
\hline | 3.7.1 & 0.00 & 52.00 & 5.50 & 11.00 & 0.015 & S-Shape \\
\hline | 3.8 .1 & 0.00 & 20.00 & 1.00 & 20.00 & 1.000 & Linear \\
\hline | 3.8.2 & 20.00 & 50.00 & 1.00 & 50.00 & 1.000 & Linear \\
\hline I 4.1.1 & 0.00 & 1.00 & n.a. & n.a. & n.a. & Stepped \\
\hline | 4.2.1 & 30.00 & 0.00 & 2.50 & 15.00 & 0.050 & Concave \\
\hline | 4.3.1 & 0.00 & 30.00 & 3.00 & 5.00 & 0.025 & S-Shape \\
\hline | 4.4.1 & 10.00 & 30.00 & 0.70 & 30.00 & 2.000 & Convex \\
\hline
\end{tabular}

\section{A case study in the Arequipa Region, Peru: The Tia Maria project}

Southern Copper, a multinational leading copper mining company, is developing the Tia Maria project at the province of Islay in the Peruvian Arequipa Region. The project, which is scheduled to start operations by 2017 and foresees an initial estimated production of 120,000 tons of copper for the first year, involves the construction of a raft leaching with a capacity of $131,250 \mathrm{~m}^{3}$ across a surface of $37,500 \mathrm{~m}^{2}$. The Environmental Impact Assessment (EIA) of the Tia Maria project was approved by the Peruvian Ministry of Energy and Mines in August 2014. However, the outright rejection of the project by the community of Islay has forced Southern Copper to temporarily paralyze the project in order to clarify a series of issues with the inhabitants of the area.

The aim of the case study is the application of SIRSDEC to the raft leaching project in Peru, taking into account information of the approved EIA to appraise its sustainability. According to the United Nations specifications (UN-Habitat, 2015), countries with a Human Development Index (HDI) below 0.8 are considered as Developing Countries. The 2015 HDI of Peru is 0.734 , which justifies the use of SIRSDEC to assess this project from the perspective of the TBL.

As a result of the application of mandatory Southern Copper policies and standards, every project developed by the firm must be aligned with ISO 9001 and 14001 specifications (C1.1) and include Project Sustainability Management and Sustainability Risk Management plans (C1.2 and C1.3). In addition, Sustainable procurement and Inspection \& Auditing plans (C1.4 and C1.5) are also implemented in all projects carried out by this company. Lessons learned from the past are logged and distributed among the organization staff to decrease the repetition of the same mistakes in the future (C1.6). Consequently, all management indicators for the Tia Maria project were rewarded with 1 point each. 
The involvement of community, stakeholders and indigenous people during the project development was null. Furthermore, $35 \%$ of local population might be impacted by the project. Consequently, the value of I2.1.1, I2.2.1 and I2.4.1 was 0 . The fact that there was no disturbance to settlements, farmland area and floodplains granted 1 point to I2.4.2, 14.2.1 and 13.6.1. Company standards also demanded the assessment of local cultural heritage and economy and the equality of wages for both sexes, which rewarded I2.4.2, I2.5.1, I4.1.1 and I2.3.1 with 1 point.

The project did not envisage energy savings, use of renewables and runoff water storage, which allocated 0 points to 13.4.1, I3.4.2 and 13.5.2. The impact of the project was estimated to affect $37 \%$ of endangered species and $10 \%$ of local employment, which rewarded I3.2.1 and I4.4.1 with 0 points. $8 \%$ of impacted ecosystem area and fresh water consumption reduction granted 0.5 and 0.26 points to 13.1 .1 and 13.5 .1 , respectively. Waste production and waste recycled/reused experienced a decrease in $15 \%$ and $47 \%$, so that indicators 13.8 .1 and 13.8 .2 received 0.83 and 0.95 points each. Local materials consumption was calculated to be $12 \%$, which means a value of 0.29 for I.4.3.1. GHG emissions and Air pollutants reduction were $18 \%$ and $23 \%$, which resulted in values of 0.34 and 0.58 for indicators 13.3 .1 and I3.7.1. Table 7 summarizes the ratings and values reached by the SIRSDEC indicators for the Tia Maria project.

Table 7. Assessment of SIRSDEC Indicators for the Tia Maria project

\begin{tabular}{|c|c|c|c|}
\hline & Indicator & $\begin{array}{l}\text { Tia Maria project } \\
\text { Indicator rating }\end{array}$ & $\begin{array}{l}\text { Tia Maria project } \\
\text { indicator value }\end{array}$ \\
\hline$\left({ }^{*}\right)$ & I 1.1.1 & 1.00 & 1.00 \\
\hline$(*)$ & | 1.1 .2 & 1.00 & 1.00 \\
\hline \multirow{2}{*}{$\left({ }^{\star}\right)$} & | 1.2 .1 & 1.00 & 1.00 \\
\hline & | 1.3 .1 & 1.00 & 1.00 \\
\hline$\left({ }^{*}\right)$ & | 1.4 .1 & 1.00 & 1.00 \\
\hline \multirow[t]{3}{*}{$\left({ }^{*}\right)$} & | 1.5 .1 & 1.00 & 1.00 \\
\hline & | 1.6 .1 & 1.00 & 1.00 \\
\hline & | 1.6 .2 & 1.00 & 1.00 \\
\hline \multirow[t]{2}{*}{$\left({ }^{*}\right)$} & I 2.1.1 & Not involved & 0.00 \\
\hline & | 2.2 .1 & Not involved & 0.00 \\
\hline \multirow[t]{3}{*}{$\left({ }^{*}\right)$} & | 2.3.1 & $100 \%$ & 1.00 \\
\hline & 2.4 .1 & $35 \%$ & 0.00 \\
\hline & 12.4 .2 & 0.00 & 1.00 \\
\hline & 12.5.1 & 1.00 & 1.00 \\
\hline \multirow[t]{2}{*}{$\left({ }^{*}\right)$} & I 3.1 .1 & $8 \%$ & 0.50 \\
\hline & 3.2 .1 & $37 \%$ & 0.00 \\
\hline \multirow{2}{*}{$\left({ }^{\star}\right)$} & I 3.3 .1 & $18 \%$ & 0.34 \\
\hline & | $3 . \overline{4} .1$ & $0 \%$ & 0.00 \\
\hline 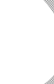 & | 3.4 .2 & $0 \%$ & 0.00 \\
\hline \multirow{2}{*}{$\begin{array}{l}(*) \\
\left({ }^{*}\right)\end{array}$} & | 3.5.1 & $8 \%$ & 0.26 \\
\hline & | 3.5 .2 & $0 \%$ & 0.00 \\
\hline \multirow[t]{4}{*}{$(*)$} & | 3.6 .1 & $0 \%$ & 1.00 \\
\hline & | 3.7 .1 & $23 \%$ & 0.58 \\
\hline & | 3.8.1 & $15 \%$ & 0.83 \\
\hline & I 3.8 .2 & $47 \%$ & 0.95 \\
\hline \multirow[t]{3}{*}{$\left({ }^{*}\right)$} & I 4.1.1 & 1.00 & 1.00 \\
\hline & | 4.2.1 & $0 \%$ & 1.00 \\
\hline & | 4.3.1 & $12 \%$ & 0.29 \\
\hline$\left({ }^{*}\right)$ & | 4.4.1 & $10 \%$ & 0.00 \\
\hline \multicolumn{2}{|c|}{ SIRSDEC score } & & 69.66 \\
\hline
\end{tabular}

$\left(^{*}\right)$ Mandatory indicators

Even though the SIRSDEC score obtained was 69.66 (Silver), which is over 63.00 (Pass), the project did not fulfil some mandatory indicators such as I2.1.1, I3.1.1, I3.3.1, I3.5.2 and 14.4.1. Consequently, the Tia Maria project did not reach the minimum score required to pass the SIRSDEC evaluation. Moreover, the values for indicators I2.4.1, I3.2.1, I3.4.1, I3.4.2 and 14.2.1 were out of the system thresholds. 
Some actions were suggested to be implemented in the project to fulfill the principles being sought by SIRSDEC and reach the Pass level of achievement, including the reduction of current social rejection. These actions intended to enhance the involvement of social stakeholders and indigenous community, in order to increase the knowledge about the project among population throughout a broad information campaign and periodic meetings with the community. The main concern of inhabitants is the negative impact of mining project on farmlands, because agriculture is their primary source of income. In this sense, the majority of manpower might be appointed among local inhabitants during the construction stage and remain during the operation of the mine. Hence, the rise of the local employment ratio up to $25 \%$ of population could contribute to mitigate economic concerns.

The raft leaching project would also incorporate additional design improvements to prevent from the break and overflow of the infrastructure, which might have very negative impacts on both ecosystem and biodiversity. These changes also would reduce GHG and air pollutants emissions. The construction of a runoff water tank would enable the reduction of fresh water consumption. Furthermore, the installation of new photovoltaic panels would contribute to saving energy and increasing the use of renewables. Table 8 includes the proposed actions and their impact in the re-assessment of the affected indicators. The implementation of these new measures would result in a SIRSDEC score for the Tia Maria project of 95.10 (Gold), including the fulfillment of all mandatory indicators and keeping within the ranges established by the framework.

Table 8. Re-assessment of affected SIRSDEC indicators

\begin{tabular}{|c|c|c|c|c|c|}
\hline Indicator & Action & $\begin{array}{c}\text { Initial } \\
\text { indicator rating }\end{array}$ & $\begin{array}{c}\text { Final } \\
\text { indicator rating }\end{array}$ & $\begin{array}{c}\text { Initial } \\
\text { indicator value }\end{array}$ & $\begin{array}{c}\text { Final } \\
\text { indicator value }\end{array}$ \\
\hline$\left({ }^{*}\right) \mid 2.1 .1$ & Information Campaign and meetings & Not involved & Involved & 0.00 & 1.00 \\
\hline 12.2.1 & Information Campaign and meetings & Not involved & Involved & 0.00 & 1.00 \\
\hline |2.4.1 & $\begin{array}{l}\text { Design improvements \& increase of } \\
\text { local employment rate }\end{array}$ & $35 \%$ & $23 \%$ & 0.00 & 0.01 \\
\hline$\left({ }^{*}\right)$ |3.1.1 & Design improvements & & $0 \%$ & 0.50 & 1.00 \\
\hline |3.2.1 & Design improvements & $37 \%$ & $23 \%$ & 0.00 & 0.03 \\
\hline (*) 13.3.1 & Design improvements & $18 \%$ & $40 \%$ & 0.34 & 1.00 \\
\hline |3.4.1 & Design improvements & $0 \%$ & $11 \%$ & 0.00 & 0.07 \\
\hline 13.4.2 & Photovoltaic panels & $0 \%$ & $14 \%$ & 0.00 & 0.33 \\
\hline$\left(^{*}\right) \mid 3.5 .1$ & Runoff water tank construction & $8 \%$ & $15 \%$ & 0.26 & 0.75 \\
\hline$(*) \mid 3.5 .2$ & Runoff water tank construction & $0 \%$ & $20 \%$ & 0.00 & 0.77 \\
\hline |3.7.1 & Design improvements & $23 \%$ & $29 \%$ & 0.58 & 0.96 \\
\hline |4.4.1 & local employment priority & $10 \%$ & $25 \%$ & 0.00 & 0.91 \\
\hline
\end{tabular}

Although Table 5 shows a set of consensual weights which is considered valid for all developing countries because it comes from the responses provided by worldwide specialists in sustainability, sensitivity analysis was conducted to determine the response of SIRSDEC when some of these weights are altered. In particular, a new scenario was designed to replicate the average distribution of weights considered in existing sustainable infrastructure rating systems in developed countries: CEEQUAL, Infrastructure Sustainability (IS) and Envision Superior. According to Diaz-Sarachaga et al. (2016b), the economic, environmental and social requirements in these systems reached average weights of $0.103,0.682$ and 0.215 , respectively. The re-assessment of the Tia Maria project using SIRSDEC after modifying the weights in Table 5 according to these values resulted in a score of 54.16, which is under 63.00 (Pass) and, by extension, under the score obtained for the weighting scenario determined from the opinions provided by the experts: 69.66 (Silver). This fact highlighted the need to increase the importance of social and economic aspects in the assessment of infrastructure projects in developing countries to obtain a realistic valuation of their contribution to sustainability, as pointed out in Diaz-Sarachaga et al. (2016a, 2016b).

\section{Conclusions}

Massive international investments on sustainable development in poorer countries demand effective guidelines and frameworks to ensure the achievement of sustainable goals. Assessment tools require the development of customized 
indicators from international development agencies that emphasize the role of infrastructure as a key driver for sustainable development. This article presents the step-by-step application of the methodology created for the design of the Sustainable Infrastructure Rating System for Developing Countries (SIRSDEC) through an infrastructure project located in the Arequipa Region (Peru).

The analysis of the responses in the questionnaires sent to intentional experts revealed that they valued positively the creation of a sustainable infrastructure rating system focused on developing countries. The distribution of weights for the requirements resulted in an almost complete balance between the three pillars of sustainable development, with a slight predominance of the social dimension $(32.4 \%)$ over the environmental $(28.9 \%)$ and economic $(24.7 \%)$ aspects. This was one of the aims of SIRSDEC in comparison with existing infrastructure rating systems, which are biased towards environmental concerns. The experts also welcomed the initiative of including management as the fourth requirement to strengthen the linkage between the three cornerstones forming the Triple Bottom Line.

The AHP method was used to transform the linguistic opinions provided by the experts into numerical pairwise comparisons. The inconsistences found in the returned questionnaires were adjusted using the Generalized Reduced Gradient algorithm, whilst the subsequent set of consistent pairwise comparisons was aggregated into a consensual vector of weights according to the similarity of thought among the experts. The application of the Integrated Value Model for Sustainable Assessment (MIVES) enabled the characterization of indicators and their standardization into a dimensionless value index using value functions. Some data from existing sustainable infrastructure rating tools were considered to establish the ranges that delimit these functions, due to the lack of statistics focused on developing countries.

The results of the case study showed the relevance of social and economic issues over environmental concerns in developing countries. SIRSDEC identified key indicators, which were initially neglected by the construction company, to promote a new approach for the community and unblock the Tia Maria project. Despite it did not initially achieve the minimum SIRSDEC requirements to be considered sustainable, its re-assessment through the proposal of several actions mainly focused on social and economic aspects enabled the achievement of sustainable objectives. Furthermore, the re-assessment of the project using the average weights used in current sustainable infrastructure rating systems resulted in an undervaluation of its contribution to sustainability, in comparison with the initial scenario based on the weights obtained from the experts. Therefore, environmental issues contributed less to the score than social and economic matters, which indicates that the influence of the latter on sustainability increases in developing countries.

This research ratifies SIRSDEC as an effective sustainable infrastructure rating system oriented to developing countries under the balanced consideration of the three principles of sustainability. However, although this paper is a promising starting point to demonstrate the usefulness of SIRSDEC to assess the contribution of infrastructure projects to sustainable development, further research should consider the inclusion of new sustainability indicators from international agencies and multilateral banks, in order to better represent the economic and social priorities of poorer countries and facilitate the collection of information throughout the lifecycle of this kind of projects. Moreover, the specifics of some particular locations might require a customization of the weights assigned to the elements forming SIRSDEC, in case there are any special reasons why some indicators must be more important than usual.

\section{References}

Abadie J and Carpentier J (1968) Generalization of the Wolfe reduced gradient method to the case of nonlinear constraints, 37-47.

Belton V and Stewart TJ (2002) Multiple criteria decision analysis: An integrated approach. Kluwer Academic. Boston. CEEQUAL (2015). CEEQUAL. Retrieved from http://www.ceequal.com on $28^{\text {th }}$ November 2015.

Chandratilake SR, Dias WPS (2013) Sustainability rating systems for buildings: comparisons and correlations. Energy 59, 22-28.

Diaz-Sarachaga JM, Jato-Espino D and Castro-Fresno D (2016a) Methodology for the development of a new Sustainable Infrastructure Rating System for Developing Countries (SIRSDEC). Environmental Science \& Policy. Under review. 
Diaz-Sarachaga JM, Jato-Espino D, Alsulami B and Castro-Fresno D (2016b) Evaluation of existing sustainable infrastructure rating systems for their application in developing countries. Ecological Indicators 71, 491-502.

ETCG (2015) Integrated Value Model for Sustainable Assessments (MIVES). Retrieved from https://www.etcg.upc.edu/pri/mives on 4th November 2015.

[19] EU, European Commission (2016) Environment. Retrieved from http://ec.europa.eu/environment/air/pollutants/ceilings.htm on 12th January 2016.

Gibberd J (2005) Assessing sustainable buildings in developing countries - the sustainable building assessment tool (SBAT) and the sustainable building lifecycle (SBL). Proceedings of the world sustainable building conference. Pages 1605-12.

Greco S, Matarazzo B and Ehrgott M (2005) Multiple criteria decision analysis: State of the art surveys. Springer Verlag. London.

Grubbs FE (1950) Sample criteria for testing outlying observations. Annals of Mathematical Statistics 21(1), 27-58.

Hiremath RB, Balachandra P, Kumar B, Bansode SS. and Murali J (2013). Indicator-based urban sustainability- a review. Energy for Sustainable Development 17(6), 555-563.

IAP2 (2016) International Association for Public Participation. Retrieved from https://www.iap2.org on $12^{\text {th }}$ February 2016.ILO, International Labour Office (2015) Global wage report 2014/2015. Wages and income inequality. http://www.ilo.org/wcmsp5/groups/public/@dgreports/@dcomm/@publ/documents/publication/wcms 324678.pdf

ISCA (2012). IS (Infrastructure Sustainability Rating Tool). Retrieved from http://www.isca.org.au on $12^{\text {th }}$ December 2015.

ISI (2012). ENVISION. Retrieved from http://www.sustainableinfrastructure.org on $23^{\text {rd }}$ November 2015.

Jato-Espino D, Indacoechea-Vega I, Gaspar L and Castro-Fresno D (2016) Decision support model for the selection of asphalt wearing courses in highly-trafficked roads. Expert Systems with Applications. Under review.

Libovich A (2005) Assessing green building for sustainable cities. Proceedings of the world sustainable building conference. Tokyo. p. 1968-71.

Saaty TI (1980) The Analytic hierarchy process: Planning, priority setting, resource allocation. McGraw-Hill.

Saaty TI (1990) How to make a decision: the analytic hierarchy process. European Journal of Operational Research, 48 (1), 9-26.

UN, United Nations (1992) Agenda 21 Contents. United Nations Conference on Environment \& Development. Rio de Janeiro. 3 to 14 June 1992. Retrieved from https://sustainabledevelopment.un.org/content/documents/Agenda21.pdf UN, United Nations (2000) UN Millennium Declaration. Millennium Summit. www.unmilleniumproject.org

UN, United Nations (2015) Transforming our World: the 2030 Agenda for Sustainable Development. Retrieved from http://www.un.org/ga/search/view doc.asp?symbol=A/RES/70/1\&Lang=E on 10

UN-Habitat, United Nations Habitat (2015) E-Governance and Urban Policy Design in Developing Countries. Retrieved from http://unhabitat.org/books/e-governance-and-urban-policy-design-in-developing-countries/ on $17^{\text {th }}$ January 2016. 